\title{
Privatrechtliche Schadensersatzansprüche zur Durchsetzung des Unionsrechts am Beispiel der Schadensersatzrichtlinie 2014/104/EU
}

Von Jan Oster, Leiden*

\section{Inhaltsübersicht}

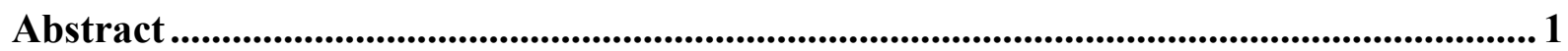

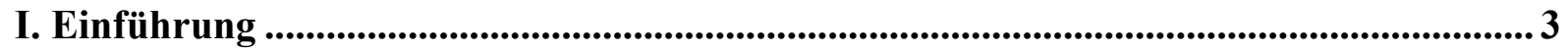

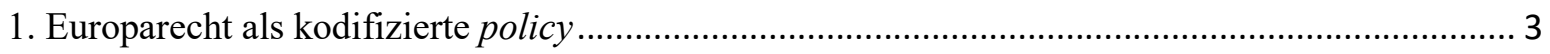

2. Verhaltenssteuerung im europäischen Privatrecht ......................................................................... 5

3. Die intrinsische und die instrumentale Funktion subjektiver Rechte im Europarecht ................... 6

II. Privatrechtliche Ansprüche als Instrument zur Durchsetzung des Unionsrechts ........ 8

1. Die Antidiskriminierungs-Rechtsprechung der 1980er und 1990er Jahre .................................... 8

2. Die Rechtssachen Courage, Muñoz und Manfrediin den 2000er Jahren....................................... 9

3. Elemente und Funktionen von Ansprüchen des europäischen Privatrechts ................................. 13

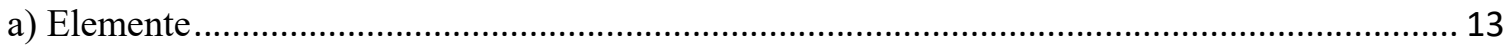

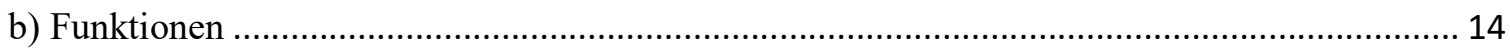

III. Das Beispiel der Schadensersatzrichtlinie 2014/104/EU ............................................ 19

1. Bekenntnis der Richtlinie zur privaten Rechtsdurchsetzung................................................... 20

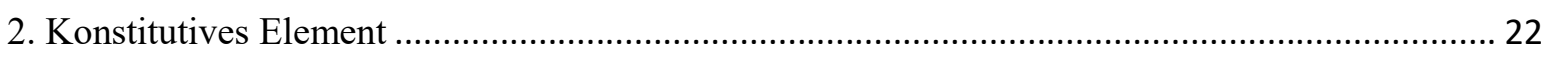

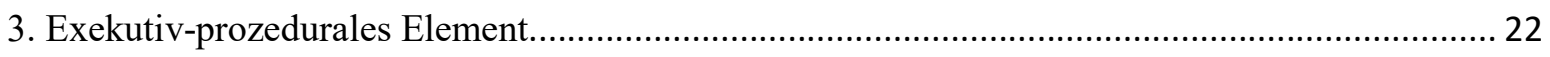

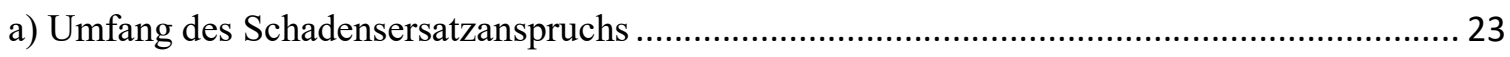

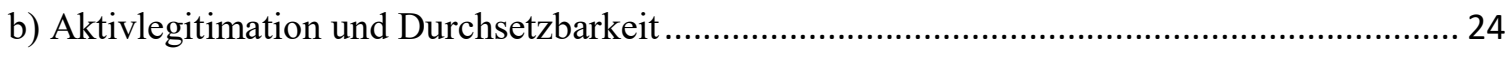

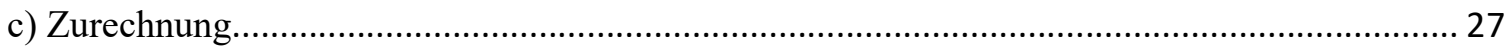

IV. Praktische Konsequenzen am Beispiel der Auslegung des $§ 33$ Abs. 3 GWB............ 28

\begin{abstract}
To what extent do claims for damages serve as an instrument to enforce EU law as such? The article argues that EU law furnishesindividual rights not only with an intrinsic, but also with an instrumental function. EU law is codified policy, serving, in particular, the establishment of the internal market. As a consequence, the conceptof individual rights as a tool to pursue and enforce certain policy aimsis much more prominent under EU law than under German

Priv.-Doz. Dr. Jan Oster, LL.M. (Berkeley), ist Assistant Professor for EU Law and Institutions an der Universität Leiden/Niederlande. Der Beitrag beruht auf dem Habilitationsvortrag, den der Verfasser am Fachbereich Rechtswissenschaften der Philipps-Universität Marburg hielt.
\end{abstract}


domestic law. The article provides a theoretical and doctrinal analysis of this development against the backdrop of the theory of neo-functionalism with its prediction of a 'spillover effect' of European integration, using the EU Antitrust Damages Directive 2014/104/EU as a case study.

Inwiefern dienen Schadensersatzansprüche der Durchsetzung des EU-Rechts selbst? Der Beitrag stellt die These auf, dass subjektiven Rechten im Unionsrecht nicht nur eine intrinsische, sondern auch eine instrumentale Funktion zukommt. EU-Recht ist kodifizierte policy und dient insbesondere der Verwirklichung des Binnenmarktes. Folglich ist das Konzept subjektiver Rechte als Instrument zur Umsetzung politischer Zielsetzungen im EURecht stärker vertreten als im deutschen Recht. Der Beitrag analysiert diese Entwicklung theoretisch und dogmatisch am Beispiel der Schadensersatzrichtlinie 2014/104/EU und vor dem Hintergrund des vomNeofunktionalismus vorhergesagten „Ausstrahlens“ (spillovereffect) europäischer Integration. 


\section{Einführung}

Es ist die größte Herausforderung europawissenschaftlicher und damit auch europarechtlicher Forschung, im Besonderen das Allgemeine zu erkennen. Das Besondere, mit dem sich dieser Beitrag befasst, ist die Schadensersatzrichtlinie 2014/104/EU (nachfolgend „Schadensersatzrichtlinie“). ${ }^{1}$ Das Allgemeine, das - gleichsam auf mittlerer Abstraktionsebene - dahintersteht, ist eine Dogmatik privatrechtlicher Schadensersatzansprüche zur Durchsetzung des Unionsrechts insgesamt. Der Beitrag geht der Frage nach, inwieweit sich die Schadensersatzrichtlinie in eine allgemeine Dogmatik des europäischen Schadensrechts zur Durchsetzung des Unionsrechts einfügen lässt. Dieser deduktiv-theoretische Ansatz ist dadurch gerechtfertigt, dass privatrechtliche Ansprüche als Instrument zur Durchsetzung des Unionsrechts aus „Geist und System“ des europäischen Primärrechts und nicht aus den Rechtsordnungen der Mitgliedstaaten erwachsen. ${ }^{2}$ Methodisch lassen sie sich somit nur direkt aus dem Primärrecht selbst ableiten und nicht durch Rechtsvergleichung ermitteln. ${ }^{3}$

\section{Europarecht als kodifizierte policy}

In ihrer neofunktionalistischen Ontologie ${ }^{4}$ beruht die Europäische Union auf einem dynamischen Integrationsprozess. ${ }^{5}$ Viel stärker als nationale Verfassungen ist das europäische

1 RL2014/104/EU des EP und des Rates vom 26.11.2014 über bestimmte Vorschriften für Schadensersatzklagen nach nationalem Recht wegen Zuwiderhandlungen gegen wettbewerbsrechtliche Bestimmungen der Mitgliedstaaten und der Europäischen Union, ABl.EU 2014 L 349/1.

2 Zu dieser Differenzierung GA Trstenjak, Schlussanträge v. 20.6.2009 in der Rs. C-101/08 (Audiolux), Rn. 69 m.w.N.

3 Vgl. GA Trstenjak, Schlussanträge v. 20.6.2009 in der Rs. C-101/08 (Audiolux), Rn. 69; J. Basedow, Der Europäische Gerichtshof und das Privatrecht. Über Unsicherheiten, Allgemeine Grundsätze und die europäische Justizarchitektur, AcP 210 (2010), S. 157, 179.

4 ZurTheorie des NeofunktionalismusgrundlegendE. B. Haas, The Uniting of Europe: Political, Social and Economic Forces 1950-1957, 1958; weiterentwickeltetwadurchL. N. Lindberg, The Political Dynamics of European Economic Integration, 1963; P. C. Schmitter, A Revised Theory of Regional Integration, International Organization 24 (1970), S. 836 ff.; J. Tranholm-Mikkelsen, Neo-functionalism: Obstinate or Obsolete? A Reappraisal in the Light of the New Dynamism of the EC, Millenium: Journal of International Studies 20 (2001), S. 1 ff.; A. Stone Sweet/W. Sandholtz, European integration and supranational governance, Journal of European Public Policy 4 (1997), S. 297 ff.; A. Niemann, Explaining Decisions in the European Union, 2006.

5 Der Gedanke eines Integrationsprozesses findet sich gleich in der Präambel des EUV wieder, wo es u.a. heißt:

„ENTSCHLOSSEN, den mit der Gründung der Europäischen Gemeinschaften eingeleiteten Prozess der europäischen Integration auf eine neue Stufe zu heben, [...] 
Primärrecht deswegen final, zweck- und zielgerichtet. ${ }^{6}$ So befindet sich die EU im „Kampf“ gegen verschiedene Formen der Diskriminierung, ${ }^{7}$ sie betreibt eine normativ zielgerichtete Außenpolitik, ${ }^{8}$ und Richtlinien sind nach Art. 288 Abs. 3 AEUV für die Mitgliedstaaten „hinsichtlich des zu erreichenden Ziels verbindlich“. In den Handlungen der EU geht es folglich immer um die Umsetzung einer begrenzten politischen Zielsetzung, einer sog. policy. Das Recht ist in diesem Zusammenhang zu verstehen als ein Mittel zur Erreichung eines politischen Ziels: Europarecht ist kodifizierte policy.

Herzstück des Integrationsprozesses ist die „Verwirklichung“ des Binnenmarktes. ${ }^{9}$ Und selbst dies ist kein Selbstzweck; der politischen Ökonomie des Binnenmarktes als einer Stufe internationaler Wirtschaftsintegration ${ }^{10}$ kommt eine instrumentale Funktion zu, nämlich die Umsetzung des Schuman-Plans: Krieg „undenkbar“ und „materiell unmöglich“ zu machen. ${ }^{11}$ Europäisches Privatrecht, das auf die Binnenmarkt-Rechtsetzungskompetenz des Art. 114 Abs. 1 Satz 2 AEUV $^{12}$ gestützt wird, darf nach der Rechtsprechung des EuGH nicht bloß Vorschriften angleichen, sondern es muss bezwecken, die Voraussetzungen für die Errichtung und das Funktionieren des Binnenmarktes zu „verbessern“. ${ }^{13}$ Diese Verbesserung geschieht jeweils punktuell, denn nach dem Prinzip der begrenzten Einzelermächtigung ${ }^{14}$ hat die EU die

IM HINBLICK auf weitere Schritte, die getan werden müssen, um die europäische Integration voranzutreiben, $[\ldots]^{“}$.

6 So heißt es im EUV in Art. 1 Abs. 1: „zur Verwirklichung ihrer gemeinsamen Ziele“, in Art. 1 Abs. 2: „Verwirklichung einer immer engeren Union“, in Art. 3 Abs. 1: „Ziel der Union ist es, den Frieden, ihre Werte und das Wohlergehen ihrer Völker zu fördern“, in Art. 3 Abs. 6: „Die Union verfolgt ihre Ziele“.

7 Vgl. Art. 19 Abs. 1 AEUV.

8 Art. 3 Abs. 5, Art. 21 EUV; grundlegend zum Konzept der „Normative Power Europe“ I. Manners, Normative Power Europe: A Contradiction in Terms?, Journal of Common Market Studies 40 (2002), S. 235 ff.

9 Art. 3 Abs. 3 EUV, Art. 26 Abs. 1, 114 Abs. 1 Abs. 1 Satz 1 und 169 Abs. 2 Buchst. a) AEUV.

10 DazuA. M. El-Agraa, General Introduction: the EU within the context of regional integration worldwide, in: El-Agraa (Hrsg.), The European Union - Economics and Policies, 9. Aufl. 2011, S. 1 ff.

11 Siehe die sog. Schuman-Erklärung vom 9.5.1950, abrufbar unter https://europa.eu/european-union/abouteu/symbols/europe-day/schuman-declaration_de. Auch dieser Gedanke findet sich in den Verträgen - etwas versteckt - wieder. In Art. 3 Abs. 3 Satz 1 ËUV heißt es lapidar: „Die Union errichtet einen Binnenmarkt.“ Der erste Absatz desselben Artikels statuiert allerdings das "Ziel der Union“ und damit auch des Binnenmarktes, „den Frieden, ihre Werte und das Wohlergehen ihrer Völker zu fördern“.

12 Die Vollendung eines einheitlichen Binnenmarktes erhielt ihr rechtliches Fundament mit der Einheitlichen Europäischen Akte von 1986 (ABl.EG 1987 L 169/1). Diese fügte dem EWG-Vertrag die Vorgängerregelung zu Art. 114 AEUV ein (den damaligen Art. 100a EWG-Vertrag), welcher als Grundlage für weite Teile der Gesetzgebung im europäischen Privatrecht diente und dient.

13 Vgl. EuGH, Rs. C-376/98 (Tabakwerberichtlinie I),ECLI:EU:C:2000:544, Slg. 2000, I-08419, Rn. 83 f.

14 Art. 5 Abs. 1 Satz 1, Abs. 2 EUV. 
Kompetenz für einzelne Privatrechtspolitiken, nicht jedoch für eine umfassende Privatrechtspolitik. $^{15}$

\section{Verhaltenssteuerung im europäischen Privatrecht}

Vor diesem Hintergrund ist auch die Verhaltenssteuerung im europäischen Privatrecht deutlich stärker ausgeprägt als im deutschen. Dies soll nicht bedeuten, dass das deutsche Privatrecht keine verhaltenssteuernden Elemente birgt. ${ }^{16}$ Das europäische Privatrecht ist aber bereits kraft Natur der Sache verhaltenssteuernd. Diese Verhaltenssteuerung erfolgt zumeist offen, nämlich dann, wenn sich jemand wegen eines Verstoßes gegen das Unionsrecht Ansprüchen ausgesetzt sieht; diese Form der Verhaltenssteuerung ist Gegenstand der weiteren Ausführungen. Die Verhaltenssteuerung kann allerdings auch verdeckt erfolgen, indem Betroffene unmittelbar rechtliche Nachteile erleiden, weil sie bestimmten Pflichten nicht nachkommen. ${ }^{17}$

Der verhaltenssteuernde Charakter von Ansprüchen des europäischen Privatrechts ist eine Folge der Konzeption subjektiver Rechte im Europarecht insgesamt. Indem der EuGH in van Gend\& $\operatorname{Loos}^{18}$ anerkannte, dass das Gemeinschaftsrecht dem Einzelnen subjektive Rechte gewährt, stellte er zwar die Freiheit des einzelnen Marktbürgers in den Mittelpunkt des Gemeinschaftsrechts. ${ }^{19}$ Damit einher ging aber zugleich die Indienstnahme des Individuums zu den Zwecken der Durchsetzung des Europarechts selbst. ${ }^{20}$

15 Statt vieler B. Heiderhoff, Europäisches Privatrecht, 4. Aufl. 2016, Rn. 13 ff.

16 Dazu nur G. Wagner, Prävention und Verhaltenssteuerung durch Privatrecht - Anmaßung oder legitime Aufgabe?, AcP 206 (2006), S. 352 ff.

17 So erhält der Unternehmer gemäß Art. 14 Abs. 2 Satz 2 Verbraucherrechterichtlinie 2011/83/EU keinen Ersatz für den Wertverlust seiner Waren, wenn er den Verbraucher nicht ordnungsgemäß über sein Widerrufsrecht belehrte. Für die Zusendung unbestellter Waren hat der Unternehmer gemäß Art. 27 Satz 1 Verbraucherrechterichtlinie keinen Anspruch auf Gegenleistung. Auch entschied der EuGH in der Rechtssache Quelle, dass Art. 3 Verbrauchsgüterkaufrichtlinie 1999/44/EG so auszulegen ist, dass der Verkäufer, der ein vertragswidriges Verbrauchsgut geliefert hat, vom Verbraucher keinen Wertersatz für die Nutzung des vertragswidrigen Verbrauchsguts bis zu dessen Austausch verlangen darf. Zur Begründung führte der EuGH u.a. aus, dass ein Verkäufer, der ein vertragswidriges Verbrauchsgut liefert, seine vertragliche Verpflichtung nicht ordnungsgemäß erfüllt und „daher die Folgen dieser Schlechterfüllung tragen [muss]“(EuGH, Rs. C-404/06 (Quelle), ECLI:EU:C:2008:231, Slg. 2008, I-02685, Rn. 41).

18 EuGH, Rs. 26/62 (van Gend\& Loos), ECLI:EU:C:1963:1, Slg. 1963, 00003, S. 26.

19 C. Kohler/J.-C. Puffer-Mariette, EuGH und Privatrecht - Ein Rückblick nach 60 Jahren, ZEuP 2014, S. 696, 738.

20 C. Heinze, Schadensersatz im Unionsprivatrecht, 2017, S. 18 m.w.N.; A. Hellgardt, Regulierung und Privatrecht, 2016, S. $184 \mathrm{ff}$. 


\section{Die intrinsische und die instrumentale Funktion subjektiver Rechte im Europarecht}

Somit lassen sich zwei Funktionen subjektiver Rechte im Europarecht unterscheiden: Zum einen sind Individuen als Träger von Rechten selbst Subjekte des Binnenmarktes und nehmen idealerweise an diesem Binnenmarkt grenzüberschreitend teil. ${ }^{21}$ Dieser Gedanke ist besonders stark ausgeprägt im europäischen Verbraucherschutzrecht: Ein hohes Verbraucherschutzniveau stärkt das Vertrauen des Verbrauchers in den Binnenmarkt, setzt Anreize, grenzüberschreitend zu konsumieren, und stärkt damit mittelbar den Binnenmarkt. ${ }^{22}$ Dies wiederum unterstreicht die friedenssichernde Funktion des Binnenmarktes als solchem: Menschen, die am Nutzen eines grenzüberschreitenden Marktes partizipieren, führen keine Kriege gegeneinander. Dieser Aspekt wird nachfolgend als die intrinsische Funktion subjektiver Rechte im Unionsrecht bezeichnet.

Zum anderen bedeutet die unmittelbare Anwendbarkeit des EU-Rechts für Individuen, dass diese durch die Geltendmachung von Rechten Verstöße gegen Pflichten aus dem EU-Recht beseitigen bzw. verhindern und so zur europäischen Integration beitragen. Durch die unmittelbare Anwendbarkeit unionsrechtlicher Bestimmungen wird also deren praktische Wirksamkeit gefördert. ${ }^{23}$ In van Gend\& Loos wies der EuGH auf folgendes hin: „Die Wachsamkeit der an der Wahrung ihrer Rechte interessierten Einzelnen stellt eine wirksame Kontrolle dar, welche die durch die Kommission und die Mitgliedstaaten [...] ausgeübte Kontrolle ergänzt.“24 In Anlehnung an US-amerikanische Terminologie ${ }^{25}$ wird hierfür häufig der Begriff des private enforcement verwendet; um konzeptionelle Missverständnisse zu vermeiden erscheint es indessen vorzugswürdig, in Gegenüberstellung zur intrinsischen

21 Siehe EuGH, Rs. 15/81 (Gaston), ECLI:EU:C:1982:135, Slg. 1982，01409，Rn. 33: „Der Begriff Gemeinsamer Markt - so wie ihn der Gerichtshof in ständiger Rechtsprechung herausgearbeitet hat - stellt ab auf die Beseitigung aller Hemmnisse im innergemeinschaftlichen Handel mit dem Ziele der Verschmelzung der nationalen Märkte zu einem einheitlichen Markt, dessen Bedingungen denjenigen eines wirklichen Binnenmarktes möglichst nahekommen. Es ist wichtig, daß die Vorteile dieses Marktes über den berufsmäßigen Handel hinaus auch Privatleuten zugute kommen, wenn sie grenzüberschreitende wirtschaftliche Transaktionen durchführen.“

22 B. Heiderhoff(Fn. 15), Rn. 191 und 229. Vgl. Erwägungsgründe 4 und 5 der Verbrauchsgüterkaufrichtlinie 1999/44/EG, Erwägungsgrund 8 der Verbraucherkreditrichtlinie 2008/48/EG, Erwägungsgründe 3 und 5 der Finanzdienstleistungs-Fernabsatz-Richtlinie 2002/65/EG sowie Erwägungsgründe 5 und 6 der Verbraucherrechterichtlinie 2011/83/EU.

23 C. Kohler/J.-C. Puffer-Mariette(Fn. 19), S. 729; M. Ebers, Rechte, Rechtsbehelfe und Sanktionen im Unionsprivatrecht, 2016, S. 123 f., 136.

24 EuGH, Rs. 26/62 (van Gend \& Loos), ECLI:EU:C:1963:1, Slg. 1963, 00003, S. 26.

25 SieheD. Poelzig, NormdurchsetzungdurchPrivatrecht, 2012, S. 51 ff.; A. Stephan, Does the EU's drive for private enforcement of competition law have a coherent purpose?, University of Queensland Law Journal 37 (2018), S. 153, $155 \mathrm{ff}$. 
Funktion den Begriff derinstrumentalenFunktion subjektiver Rechte zu verwenden. Hierzu rechnen beispielsweise auch Staatshaftungsansprüche kraft Unionsrechts. ${ }^{26}$

Diebeiden Funktionen subjektiver Rechte - die intrinsische und die instrumentale - sind zwar überlappend, aber dennoch voneinander $\mathrm{zu}$ unterscheiden. Die intrinsische Funktion subjektiver Rechte folgt bereits aus dem Wesen subjektiver Rechte und ist daher bei Lichte besehen eine tautologische Erkenntnis. Die instrumentale Funktion subjektiver Rechte ist demgegenüber eine besondere Ausprägung des von der Theorie des Neofunktionalismus vorhergesagten spillover-Effekts europäischer Integration. ${ }^{27}$ Der Begriff des ,spillover “ bringt den Gedanken der irreversiblen Fortentwicklung zum Ausdruck und impliziert zugleich das Konzept nicht beabsichtigter Folgeentwicklungen (unintendedconsequences) als Motor der Integration. ${ }^{28}$ Van Gend\& Loos und danachCosta gegen ENEL sind als „spillover" in einer Zeit zu identifizieren, in der der Neofunktionalismus scheinbar an Erklärungskraft verlor: Im damaligen Ministerrat der EWG bestand das Erfordernis der Einstimmigkeit, was später - im Jahr 1965 - Frankreichs „Politik des leeren Stuhls“ ermöglichte. Seit van Gend\& Loos vertraut das Europarecht somit darauf, dass IndividualinteressenKräftefreisetzen, die durch die Mitgliedstaaten und durch die supranationalen Institutionen allein häufig nicht entfaltet werden können. ${ }^{29}$ Waren es bis zur Entscheidung van Gend\& Loos neben der Europäischen Kommission nur die sechs Mitgliedstaaten, die die Grundfreiheiten des EWG-Vertrag geltend machen konnten, so waren es nach van Gend\& Loos ca. 180 Millionen Anspruchsberechtigte. Dies wiederum bestätigt neben dem neofunktionalistischen Erklärungsmodell auch neoinstitutionalistische Ansätze. ${ }^{30}$ Die instrumentale Funktion subjektiver Rechte gewährleistet nämlich nicht nur die dezentrale Durchsetzung des Europarechts, sondern stärkt

26 Siehe EuGH, verb. Rs. C-6/90 und C-9/90 (Francovich), ECLI:EU:C:1991:428, Slg. 1991, I-05357, Rn. 33; verb. Rs. C-46/93 und C-48/93 (Brasserie du pechêur und Factortame), ECLI:EU:C:1996:79, Slg. 1996, I01029,Rn. 39. DazustattvielerT. Lock, Is private enforcement of EU law through state liability a myth? An assessment 20 years after Francovich, Common Market Law Review 49 (2012), S. 1675 ff.

27 Diese Annahme ist zunächst nicht unproblematisch, da der Neofunktionalismus prognostizierte, dass sich die europäische Integration nicht durch Privatpersonen, sondern durch supranationale Institutionen, d.h. im Wege des öffentlichen Rechts, verwirklichen würde. Der Neofunktionalismus ist dadurch jedoch nicht widerlegt, sondern bedarf lediglich einer kleineren Korrektur. Bemerkenswert ist nämlich, dass die instrumentale Funktion subjektiver Rechte nicht durch populäre Bewegungen, sondern durch die Rechtsprechung des EuGH, der supranationalen Institution schlechthin, geschaffen wurde.

28 Ausführlich zu den Formen des spillover (funktionaler, politischer und kultivierter spillover) J. TranholmMikkelsen(Fn. 4), S. 1 ff.

29 G. Wagner (Fn. 16), S. 399; D. Poelzig(Fn. 25), S. 136 f.; M. Ebers(Fn. 23), S. 97 ff.; J.-U. Franck, Marktordnung durch Haftung, 2016, S. 172 f.; C. Heinze(Fn. 20), S. 18 f.

30 Zum Ansatz des Neoinstitutionalismus $P$. Pierson, The Path to European Integration: A Historical Institutionalist Analysis, Comparative Political Studies 29 (1996), S. 123 ff.; M. A. Pollack, The New Institutionalisms and European Integration, in: Wiener/Diez (Hrsg.), European Integration Theory, 2. Aufl. 2009, S. $125 \mathrm{ff}$. 
auch die Bedeutung des EuGH selbst - ein Nebeneffekt, der dem Gerichtshof sicher nicht unerwünscht ist.

\section{Privatrechtliche Ansprüche als Instrument zur Durchsetzung des Unionsrechts}

Privatrechtliche Ansprüche lassen sich nahtlos in diesen Rahmen einfügen. Die unmittelbare Anwendung von Unionsrechtsbestimmungen im horizontalen Verhältnis ist eine Konsequenz der unmittelbaren Wirkung des Unionsrechts, wie der EuGH sie in van Gend\& Loos etablierte. ${ }^{31}$ Auch privatrechtliche Ansprüche können ein regulatorisches Instrument sein, um Europarecht durchzusetzen. Sie sind daher nichts anderes als eine Form der governance.

\section{Die Antidiskriminierungs-Rechtsprechung der 1980er und 1990er Jahre}

Erste Ansätze für die instrumentale Funktion subjektiver Rechte lassen sich in der Rechtsprechung des EuGH aus den 1980er und 1990er Jahren zu geschlechtsbezogenen Benachteiligungen erkennen. Gemäß Art. 6 der Richtlinie 76/207/EWG mussten die Mitgliedstaaten gewährleisten, dass ,jeder, der sich wegen Nichtanwendung des Grundsatzes der Gleichbehandlung [...] auf seine Person für beschwert hält, [...] seine Rechte gerichtlich geltend machen kann.“ Art. 6 schrieb daher keine bestimmte Sanktion vor, sondern überließ den Mitgliedstaaten „die Freiheit der Wahl unter den verschiedenen, zur Verwirklichung ihrer Zielsetzung geeigneten Möglichkeiten“. ${ }^{32}$ In den Rechtssachen von Colson und Kamann, Harzsowie in darauffolgenden Entscheidungen unterstrich der EuGH jedoch, dass die vorgesehene Sanktion „einen tatsächlichen und wirksamen Rechtschutz [...] gewährleisten“ und eine ,wirklich abschreckende Wirkung gegenüber dem Arbeitgeber haben“ müsse. ${ }^{33}$ Wenn sich der Mitgliedstaat für eine privatrechtliche Schadensersatzpflicht entscheide, dann müsse der Ersatzbetrag ,in einem angemessenen Verhältnis zum erlittenen Schaden

31 GA van Gerven, Schlussanträge v. 27.10 .1993 in der Rs. C-128/92 (Banks), Rn. 43; C. Kohler/J.-C. PufferMariette (Fn. 19), S. 728 und 734.

32 EuGH, Rs. 14/83 (von Colson und Kamann), ECLI:EU:C:1984:153, Slg. 1984, 01891, Rn. 18; Rs. 79/83 (Harz), ECLI:EU:C:1984:155, Slg. 1984, 01921, Rn. 18; s.a. Rs. C-177/88 (Dekker), ECLI:EU:C:1990:383, Slg. 1990, I-03941, Rn. 23; Rs. C-271/91 (Marshall), ECLI:EU:C:1993:335, Slg. 1993, I-04367, Rn. 23; Rs. C-180/95 (Draehmpael), ECLI:EU:C:1997:208, Slg. 1997, I-02195, Rn. 24.

33 EuGH, Rs. 14/83 (von Colson und Kamann), ECLI:EU:C:1984:153, Slg. 1984, 01891, Rn. 23; Rs. 79/83 (Harz), ECLI:EU:C:1984:155, Slg. 1984, 01921, Rn. 23; Rs. C-177/88 (Dekker), ECLI:EU:C:1990:383, Slg. 1990, I-03941, Rn. 23; Rs. C-271/91 (Marshall), ECLI:EU:C:1993:335, Slg. 1993, I-04367, Rn. 24. 
stehen“. ${ }^{34}$ Von Colson und Kamann und Harz basierten somit aus einer Mischung aus Kompensations- und Präventionsgedanken: Während sich der Kompensationsgedanke in der Betonung des „angemessenen Verhältnis[ses] zum erlittenen Schaden“ wiederfindet, hat der Formel von der „,wirklich abschreckenden Wirkung“ Präventionsfunktion.

$\mathrm{Zu}$ beachten ist aber, dass eine privatrechtliche Schadensersatzpflicht in einem Arsenal möglicher Sanktionsinstrumente alternativ zu anderen stand, z.B. zu öffentlich-rechtlicher Regulierung oder Strafrecht. ${ }^{35}$ Privatrechtliche Ansprüche allgemein und Schadenersatz im Besonderen waren hier nur ein mögliches Mittel zur Durchsetzung des Europarechts, aber noch nicht ein zwingendes Mittel. Der Grundsatz „ubiius, ibiremedium“ war und ist auf europarechtlicher Ebene insofern eingeschränkt, als Recht und Rechtsbehelf grundsätzlich nicht durch denselben Normgeber geregelt werden. ${ }^{36}$ Entscheidend für die Antidiskriminierungsrechtsprechung war allein, dass der Rechtsbehelf „hinreichend wirksam“ war, um das Ziel der Richtlinie zu erreichen. Grundlage der Rechtsprechung war somit der Effektivitätsgrundsatz, wie er nunmehr in Art. 4 Abs. 3 EUV kodifiziert ist.

\section{Die Rechtssachen Courage, Muñoz und Manfrediin den 2000er Jahren}

Der EuGH vollendete die Instrumentalisierung privatrechtlicher Ansprüche als Mittel zur Durchsetzung des Europarechts erst in den 2000er Jahren. ${ }^{37}$ Im Unterschied zur Antidiskriminierungsrechtsprechung erkannte der EuGH in diesen Entscheidungen nur noch bestimmte privatrechtliche Rechtsfolgen als hinreichend effektiv an, nämlich Schadensersatzansprüche wegen Verstößen gegen das Kartellrecht in Courage ${ }^{38}$ und

34 EuGH, Rs. 14/83 (von Colson und Kamann), ECLI:EU:C:1984:153, Slg. 1984, 01891, Rn. 23; Rs. 79/83 (Harz), ECLI:EU:C:1984:155, Slg. 1984, 01921, Rn. 23; bestätigt in Rs. C-271/91 (Marshall), ECLI:EU:C:1993:335, Slg. 1993, I-04367, Rn. 26; Rs. C-180/95 (Draehmpael), ECLI:EU:C:1997:208, Slg. 1997, I-02195, Rn. 27.

35 G. Wagner (Fn. 16), S. 393.

36 C. Heinze (Fn. 20), S. 8.

37 Zuvor hatte bereits Generalanwalt van Gerven in der Rechtssache Banks privatrechtliche Schadensersatzansprüche auf gemeinschaftsrechtlicher Grundlage bejaht; in seiner anschließenden Entscheidung ging der EuGH auf diese Frage jedoch nicht ein, da er bereits die unmittelbare Wirkung der einschlägigen Vorschriften des EGKS-Vertrages verneinte: EuGH, Rs. C-128/92 (Banks), ECLI:EU:C:1994:130, Slg. 1994, I-01209, Rn. 19.

38 EuGH, Rs.C-453/99 (Courage/Crehan), ECLI:EU:C:2001:465, Slg. 2001, I-06297. 
Manfred $i^{39}$ sowie einen Unterlassungsanspruch in $M u \tilde{n} o z^{40}$ wegen Verstoßes gegen Verordnungen über die gemeinsame Marktorganisation für Obst und Gemüse. ${ }^{41}$

Der EuGH führte in Courage aus, dass ein Schadensersatzanspruch „die Durchsetzungskraft der gemeinschaftlichen Wettbewerbsregeln“ erhöhe und geeignet sei, von „Verhaltensweisen abzuhalten, die den Wettbewerb beschränken oder verfälschen können." Daher könnten Schadensersatzklagen ,wesentlich zur Aufrechterhaltung eines wirksamen Wettbewerbs in der Gemeinschaft beitragen. “42In Muñoz ergänzte der EuGH, dass die volle Wirksamkeit der einschlägigen gemeinschaftsrechtlichen Regelung voraussetze, dass deren Beachtung im Wege eines Zivilprozesses durchgesetzt werden kann, den ein Wirtschaftsteilnehmer gegen einen Konkurrenten anstrengt. ${ }^{43}$ Klagen von Konkurrenten vor nationalen Gerichten seien „besonders geeignet, wesentlich zur Sicherung eines lauteren Handels und der Markttransparenz in der Gemeinschaft beizutragen. “44

Der EuGH hat daher nicht bloß den im Antidiskriminierungsrecht entwickelten Ansatz auf das Wettbewerbsrecht übertragen, sondern einen Paradigmenwechsel vollzogen.Zwar trifft es $\mathrm{zu}$, dass sowohl der antidiskriminierungsrechtliche als auch der wettbewerbsrechtliche Schadensersatzanspruch auf dem Effektivitätsgrundsatz (effetutile, Art. 4 Abs. 3 EUV) beruhen. Die Antidiskriminierungsrechtsprechung machte allerdings keine Vorgaben hinsichtlich eines bestimmten Rechtsbehelfs. Der ihr zugrunde liegende Effektivitätsgrundsatz war daher nur „negativ“, 45 indem er nicht-effektive Rechtsbehelfe verbot. Hiervon zu

39 EuGH, verb. Rs. C-295/04 bis C-298/04 (Manfredi), ECLI:EU:C:2006:461, Slg. 2006, I-06619.

40 EuGH, Rs. C-253/00 (Muñoz), ECLI:EU:C:2002:497, Slg. 2002, I-07289.

41 Verordnungen (EWG) Nr. 1035/72 des Rates vom 18.5.1972 und (EG) Nr. 2200/96 des Rates vom 28.10.1996 über eine/die gemeinsame Marktorganisation für Obst und Gemüse (ABl.EG 1972 L 118/1 und ABl.EG 1996 L 297/1).

42 EuGH, Rs. C-453/99 (Courage/Crehan), ECLI:EU:C:2001:465, Slg. 2001, I-06297, Rn. 27; verb. Rs. C295/04 bis C-298/04 (Manfredi), ECLI:EU:C:2006:461, Slg. 2006, I-06619, Rn. 91; Rs. C-360/09 (Pfleiderer), ECLI:EU:C:2011:389, Slg. 2011, I-05161, Rn. 29; Rs. C-199/11 (Otis), ECLI:EU:C:2012:684, Rn. 42; Rs. C-536/11 (Donau Chemie), ECLI:EU:C:2013:366, Rn. 23; Rs. C-557/12 (Kone), ECLI:EU:C:2014:1317, Rn. 23. Vgl. GA van Gerven, Schlussanträge v. 27.10.1993 in der Rs. C-128/92 (Banks), Rn. 44: „Das nationale Gericht kann unter diesen Umständen die verletzten gemeinschaftsrechtlichen Bestimmungen mit unmittelbarer Wirkung nur in der Weise in vollem Umfang wahren, daß die Rechte der geschädigten Partei durch Schadensersatz wiederhergestellt werden.“

43 EuGH, Rs. C-253/00 (Muñoz), ECLI:EU:C:2002:497, Slg. 2002, I-07289, Rn. 30.

44 EuGH, Rs. C-253/00 (Muñoz), ECLI:EU:C:2002:497, Slg. 2002, I-07289, Rn. 31. Auch dieser Gedanke fand sich bereits in den Schlussanträgen van Gervens: „Daneben kommt einer solchen Schadensersatzregelung eine wichtige Rolle bei einer wirksameren Gestaltung der gemeinschaftlichen Wettbewerbsbestimmungen zu, zumal die Kommission als Hüterin dieser Bestimmungen selbst einräumt, daß sie bei der Durchsetzung der Bestimmungen auf die Mitwirkung des nationalen Gerichts angewiesen ist" (GA van Gerven, Schlussanträge v. 27.10.1993 in der Rs. C-128/92 (Banks), Rn. 44; Kursivdruck im Original, Fundstellennachweise hier nicht abgedruckt).

45 So C. Heinze (Fn. 20), S. 76 ff. 
unterscheiden ist der instrumentale Gedanke der privaten Rechtsdurchsetzung, der auf einem „positiven“46 Effektivitätsgrundsatz beruht. Anders als der negative beschränkt sich der positive Effektivitätsgrundsatz nicht darauf, den von den Mitgliedstaaten gewählten Rechtsbehelf auf seine Effektivität zu überprüfen, sondern hier gibt das Europarecht den Rechtsbehelf selbst vor. In Courage, Muñoz und Manfredi hat der EuGH daher ein neues Rechtssystem geschaffen, das die Durchsetzung primär- bzw. sekundärrechtlicher Verhaltensstandards nicht nur den Behörden anvertraut, sondern ergänzend undzwingend auf private Rechtsdurchsetzung setzt. ${ }^{47}$ Bezeichnenderweise nahmen weder der EuGH noch die Generalanwälte in Courage, Muñoz und Manfredi auf die Antidiskriminierungsrechtsprechung Bezug. ${ }^{48}$

Insofern lässt sich keine Kontinuität zwischen der Rechtsprechung zur privaten Rechtsdurchsetzung in den 2000er Jahren und der Antidiskriminierungsrechtsprechung des 20. Jahrhunderts identifizieren. Stattdessen liegt nahe, dass dem EuGH der Gedanke der privaten Rechtsdurchsetzung in seiner Antidiskriminierungsrechtsprechung noch gar nicht bewusst war. Der EuGH und die europäischen Institutionen in ihrem öffentlich-rechtlichen Ansatz der 1970er und 1980er Jahre hätten verwaltungs- oder gar strafrechtliche Sanktionsinstrumente einer rein privatrechtlichen Lösung womöglich vorgezogen. So hieß es im Kommissionsentwurf zur Richtlinie auch etwas martialisch: „Da es kein Recht ohne Strafen gibt, soll den Betreffenden, die sich geschädigt fühlen, mit dieser Richtlinie die Möglichkeit garantiert werden, gerichtlich vorgehen zu können, um ihren Anspruch auf Gleichbehandlung durchzusetzen (Artikel 6)“.49 In seiner Entschließung zum Richtlinienentwurf wies das Europäische Parlament darauf hin, dass „die Wirksamkeit der vorgeschlagenen Richtlinie nicht nur von den legislativen, sondern auch von den administrativen und verfahrensmäßigen Maßnahmen und Sanktionen abhängt" - von „zivilrechtlichen Sanktionen“ war also keine Rede. ${ }^{50}$ Dies deutet sich auch in vonColson und

${ }^{46}$ C. Heinze (Fn. 20), S. 79.

47 G. Wagner (Fn. 16), S. 447; J.-U. Franck(Fn. 29), S. 265.

48 Anders GA van Gerven, Schlussanträge v. 27.10 .1993 in der Rs. C-128/92 (Banks), Rn. 54. In Manfredizitierte der EuGH zwar die Marshall-Entscheidung, allerdings nur hinsichtlich einer Einzelfrage der Zinszahlung: EuGH, verb. Rs. C-295/04 bis C-298/04 (Manfredi), ECLI:EU:C:2006:461, Slg. 2006, I-06619, Rn. 97.

49 Europäische Kommission, Vorschlag einer Richtlinie des Rates zur Verwirklichung des Grundsatzes der Gleichbehandlung von Männern und Frauen hinsichtlich des Zugangs zur Beschäftigung, zur beruflichen Bildung und zum beruflichen Aufstieg sowie in bezug auf die Arbeitsbedingungen, V/1153/74-Rev.1-D, S. 4.

50 Entschließung mit der Stellungnahme des Europäischen Parlaments zu dem Vorschlag der Kommission der Europäischen Gemeinschaften an den Rat für eine Richtlinie über die Gleichbehandlung von Männern und Frauen im Arbeitsleben (Zugang zur Beschäftigung, zur beruflichen Bildung und zum beruflichen Aufstieg sowie in bezug auf die Arbeitsbedingungen), ABl.EG 1975 C 114/14, Rn. 12. 
Kamann an, wo der EuGH davon spricht, dass zivilrechtliche Regelungen - etwa eine Verpflichtung des Arbeitgebers zur Einstellung des diskriminierten Bewerbers oder zu einer angemessenen finanziellen Entschädigung - durch eine Bußgeldregelung ,verstärkt" werden könnten. ${ }^{51}$ Der öffentlich-rechtliche Ansatz erklärt schließlich auch die Verwendung des Wortes „Sanktion“ in der Rechtsprechung des EuGH - ein Begriff, der in der jüngeren Rechtssetzung (soweit ersichtlich) nur noch im Zusammenhang mit öffentlich-rechtlichen Interventionen verwendet wird. ${ }^{52}$ Privatrechtliche Ansprüche hat der EuGH in den 1980er Jahren noch nicht als funktionelles Äquivalent zum Verwaltungsvollzug wahrgenommen. Dies geschah explizit erst in späteren Entscheidungen. ${ }^{53}$

Dass es sich bei der privaten Rechtsdurchsetzung um einen Paradigmenwechsel im Europarecht handelt, ist auch anhand des Sekundärrechts $\mathrm{zu}$ belegen. Art. 15 der Antirassismusrichtlinie 2000/43/EG und Art. 17 der Gleichbehandlungsrahmenrichtlinie 2000/78/EG - zwei Richtlinien also, die vor der Entscheidung Courage beschlossen wurden enthalten Regelungen über „Sanktionen“, die dem Muster der Antidiskriminierungsrechtsprechung des EuGH folgen. Danach obliegt es den Mitgliedstaaten, die bei Verstößen zu verhängenden Sanktionen festzulegen, wobei die gewählten Sanktionen auch Schadensersatzleistungen umfassen können, aber eben nicht müssen. Vor allem aber müssen diese Sanktionen „wirksam, verhältnismäßig und abschreckend“ sein. Weiter gehen demgegenüber Art. 8 Abs. 2 der Gleichbehandlungsrichtlinie (Güter und Dienstleistungen) 2004/113/EG und Art. 18 der Gleichbehandlungsrichtlinie (Arbeits- und Beschäftigungsfragen) 2006/54/EG - zwei Richtlinien also die nach Courage verabschiedet wurden. Diese verpflichten die Mitgliedstaaten dazu, dem Diskriminierungsopfer einen Schadensersatzanspruch gegen den Diskriminierungstäter zu gewähren, ,wobei dies auf eine abschreckende und dem erlittenen Schaden angemessene Art und Weise geschehen muss.“ Hier besteht folglich eine größere Intensität des „Harmonisierungszugriffs “65 des Europarechts auf privatrechtliche Rechtsfolgen. Das Europarecht schreibt die Rechtsfolge „Schadensersatz“ explizit vor, und dies nicht nur in seiner Ausgleichsfunktion, sondern auch in seiner Rechtsdurchsetzungsfunktion, indiziert durch das Wort ,abschreckend“. Daran ist schließlich

\footnotetext{
51 EuGH, Rs. 14/83 (von Colson und Kamann), ECLI:EU:C:1984:153, Slg. 1984, 01891, Rn. 18.

52 Siehe z.B. Erwägungsgründe 149 ff. der Datenschutz-Grundverordnung 2016/679/EU, Art. 25 Pauschalreiserichtlinie 2015/2302/EU, Art. 8 Abs. 2 Schadensersatzrichtlinie.

53 Seit EuGH, Rs. C-352/92 (Milchwerke Köln/Wuppertal e.G.), ECLI:EU:C:1994:294, Slg. 1994, I-03385, Rn. 23.

${ }^{54}$ C. Heinze (Fn. 20), S. 107.
} 
auch zu erkennen, dass es nicht zutreffend ist, die Linie zwischen bloßer Ausgleichsfunktion einerseits und Rechtsdurchsetzungsfunktion andererseits zwischen Primär- und Sekundärrecht oder zwischen Richtlinien und Verordnungen $\mathrm{zu}$ ziehen. ${ }^{55}$ Stattdessen ist der Effektivitätsgrundsatz der ausschlaggebende Faktor dafür, ob in bestimmten Fällen der Verletzung unmittelbar wirksamer Normen - ob diese nun im Primärrecht, in Verordnungen oder in (umzusetzenden) Richtlinien kodifiziert sind - eine Auswahl aus mehreren oder nur eine bestimmte Rechtsfolge, etwa ein Schadensersatzanspruch, unionsrechtskonform ist.

\section{Elemente und Funktionen von Ansprüchen des europäischen Privatrechts}

Vor dem Hintergrund dieser Beobachtung sind Ansprüche des europäischen Privatrechts nach ihren Elementen und Funktionen wie folgt zu systematisieren.

\section{a) Elemente}

Ansprüche des europäischen Privatrechts bestehen aus zwei Elementen: Einem konstitutiven Element und einem exekutiv-prozeduralen Element. ${ }^{56}$ Das konstitutive Element betrifft die Existenz eines bestimmten Anspruchs, z.B. auf Schadensersatz. Das exekutiv-prozedurale Element betrifft demgegenüber den konkreten Inhalt des Anspruchs und seine Durchsetzung im Prozess. Es schließt beispielsweise die Kriterien für die Ermittlung des Umfangs des Schadensersatzes, ${ }^{57}$ die Bestimmung des Begriffes „ursächlicher Zusammenhang“, ${ }^{58}$ das Erfordernis der vorprozessualen Einschaltung eines Schiedsrichters, ${ }^{59}$ Zugang zu Dokumenten, ${ }^{60}$ die Bestimmung der zuständigen Gerichte ${ }^{61}$ und die Verjährungsfrist ${ }^{62}$ ein.

\footnotetext{
55 So aber C. Heinze (Fn. 20), S. $106 \mathrm{ff}$.

56 In Anlehnung an W. van Gerven, Of Rights, Remedies and Procedures, Common Market Law Review 37 (2000), S. 501, 522 ff.; A. Komninos, EC Private Antitrust Enforcement, 2008, S. 173 ff.; D. Chalmers/G. Davies/G. Monti, European Union Law, 3. Aufl. 2014, S. 990 f.

57 EuGH, verb. Rs. C-295/04 bis C-298/04 (Manfredi), ECLI:EU:C:2006:461, Slg. 2006, I-06619, Rn. 92.

58 EuGH, verb. Rs. C-295/04 bis C-298/04 (Manfredi), ECLI:EU:C:2006:461, Slg. 2006, I-06619, Rn. 64; Rs. C-557/12 (Kone), ECLI:EU:C:2014:1317, Rn. 24.

59 Vgl. EuGH, Rs. C-421/05 (City Motors Groep), ECLI:EU:C:2007:38, Slg. 2007, I-00653, Rn. 34.

60 EuGH, Rs. C-536/11 (Donau Chemie), ECLI:EU:C:2013:366, Rn. 26.

61 EuGH, verb. Rs. C-295/04 bis C-298/04 (Manfredi), ECLI:EU:C:2006:461, Slg. 2006, I-06619, Rn. 72.

62 EuGH, verb. Rs. C-295/04 bis C-298/04 (Manfredi), ECLI:EU:C:2006:461, Slg. 2006, I-06619, Rn. 81.
} 


\section{b) Funktionen}

Eine Anspruchsgrundlage des europäischen Privatrechts bezweckt immer, die Rechte eines Einzelnen zu schützen (dazu aa)) und eine politische Zielsetzung der EU zu verwirklichen (dazu bb)). Darüber hinaus kann eine Anspruchsgrundlage des europäischen Privatrechts auch dazu dienen, das EU-Recht selbst durchzusetzen (dazu cc)).

aa) Kompensationsfunktion

Jede Anspruchsgrundlage des europäischen Privatrechts dient dazu, die Rechte des Einzelnen gegenüber einem anderen zu schützen, z.B. Ansprüche aus Mängelgewährleistung, aus Produkthaftung, nach der Pauschalreiserichtlinie 2015/2302/EU,usw. Bei Schadensersatzansprüchen besteht diese Funktion insbesondere darin, einen entstandenen Schaden auszugleichen (sog. Kompensationsfunktion). ${ }^{63}$ Das konstitutive Element des Schadensersatzanspruchs, also das „Ob“, ist in den Fällen der reinen Kompensationsfunktion zumeist in das Ermessen der Mitgliedstaaten gestellt. ${ }^{64}$ Das Unionsrecht kann allerdings auch Schadensersatz als zwingende Rechtsfolge vorsehen, wie dies etwa in Art. 1

${ }^{63}$ Die Genugtuungsfunktion ist im EU-Privatrecht deutlich seltener anzutreffen (dazu J.-T. Oskierski, Schadensersatz im europäischen Recht, 2010, S. 106 ff.) und kann hier daher außer Betracht bleiben. - Zur Kompensationsfunktion des Deliktsrechts etwa J. Coleman, Corrective Justice and WrongfulGain, Journal of Legal Studies 11 (1982), S. 421 ff.; E. J. Weinrib, Corrective Justice,Iowa Law Review 77 (1992), S. 403 ff.; J. J. Coleman, The Mixed ConceptionsofCorrective Justice, Iowa Law Review 77 (1992), S. 427 ff.; Coleman, Tort Law and theDemandsofCorrective Justice, Indiana Law Review 67 (1992), S. 349 ff.; M. Rohe, Gründe und Grenzen deliktischer Haftung - die Ordnungsaufgaben des Deliktsrechts (einschließlich der Haftung ohne Verschulden) in rechtsvergleichender Betrachtung, AcP 201 (2001), S. 117, 125; S. Hershovitz, Harry Potter and the Trouble with Tort Theory, Stanford Law Review 63 (2010), S. 67,106 ff.; J. Gardner, Whatis tort lawfor? Part 1: theplaceofcorrectivejustice,Law \& Philosophy 2011, S. 1 ff.; T. AlTawil, Corrective Justice and Deterrence: Can They Co-Exist?, European Journal of Legal Studies, 6 (2013), S. 109, 112 ff. Zum Ausgleichsprinzip im europäischen Schadensrecht im Besonderen W. Wurmnest, Grundzüge eines europäischen Haftungsrechts, 2003, S. 94 ff.; G. Wagner (Fn. 16), S. 395; J.-T. Oskierski, Schadensersatz im europäischen Recht, 2010, S. 84 ff.; G. Meeßen, Der Anspruch auf Schadensersatz bei Verstößen gegen EU-Kartellrecht, 2011, S. 58 ff. Siehe beispielsweise Erwägungsgrund 12 FluggastrechteVerordnung 261/2004/EG: „Das Ärgernis und die Unannehmlichkeiten, die den Fluggästen durch die Annullierung von Flügen entstehen, sollten ebenfalls verringert werden. Dies sollte dadurch erreicht werden, dass die Luftfahrtunternehmen veranlasst werden, die Fluggäste vor der planmäßigen Abflugzeit über Annullierungen $\mathrm{zu}$ unterrichten und ihnen darüber hinaus eine zumutbare anderweitige Beförderung anzubieten, so dass die Fluggäste umdisponieren können. Andernfalls sollten die Luftfahrtunternehmen den Fluggästen einen Ausgleich leisten [... $]^{\text {“. }}$

64 Ein Beispiel hierfür war Art. 6 der Gleichbehandlungsrichtlinie 76/204/EWG. Weitere Beispiele sind Art. 15 Antirassismusrichtlinie 2000/43/EG, Art. 17 Gleichbehandlungsrahmenrichtlinie 2000/78/EG sowie die Medizinprodukterichtlinie 93/42/EWG (dazu EuGH, Rs. C-219/15 (Schmitt/TÜV Rheinland), ECLI:EU:C:2017:128, Rn. 59). 
Produkthaftungsrichtlinie 85/374/EWG der Fall ist. ${ }^{65}$ Die Ausgestaltung des exekutivprozeduralen Elements ist ebenfalls den Mitgliedstaaten überlassen, solange sie die Rechtsbehelfsgarantie des Art. 19 Abs. 1 EUV und Art. 47 GRC wahren. Aber auch dies ist nicht zwingend; der europäische Normgeber kann etwa vorgeben, in welchem Umfang er eine Kompensation für angemessen hält. So kann bereits europarechtlich determiniert sein, dass der immaterielle Schaden vom Anspruch umfasst sein muss, wie dies etwa nach der Pauschalreiserichtlinie im Lichte der Entscheidung Leitner der Fall ist. ${ }^{66}$ Das Europarecht kann den Schadensersatzanspruch aber auch auf (bestimmte) materielle Schäden beschränken und die Regelung immaterieller Schäden den Mitgliedstaaten überlassen, wie Art. 9 Satz 2 Produkthaftungsrichtlinie dies vorsieht. $^{67}$ Schließlich kann der Schadens- oder Ausgleichsanspruch der Höhe nach begrenzt sein, wie etwa in Art. 7 FluggastrechteVerordnung 261/2004/EG und Art. 16 Produkthaftungsrichtlinie. Den genannten Regelungen ist aber gemeinsam, dass ein Schadensersatzanspruch regelmäßig an die Verletzung einer Schutznorm geknüpft ${ }^{68}$ und Prävention ist allenfalls ein Nebeneffekt ist. Kurz gesagt: Soweit eine Schadensersatznorm der Kompensationsfunktion dient, bestimmt der Umfang des rechtlich zugestandenen Schadens das Maß der Prävention. ${ }^{69}$

bb) Förderung einer EU-policy

Wie bereits dargestellt, muss eine Anspruchsgrundlage des europäischen Privatrechts immer auch bezwecken, ein politisches Ziel der EU - beispielsweise die Verwirklichung des Binnenmarktes - zu erreichen, da sie anderenfalls von den EU-Organen gar nicht erlassen werden dürfte. Hierin ist der neofunktionalistische Hintergrund des europäischen Privatrechts

65 Weitere Beispiele sind Art. 18 Gleichbehandlungsrichtlinie 2006/54/EG (Arbeits- und Beschäftigungsfragen), Art. 8 Abs. 2 Gleichbehandlungsrichtlinie 2004/113/EG (Güter und Dienstleistungen), Art. 13 Durchsetzungsrichtlinie 2004/48/EG, Art. 8 Urheberrechts-Richtlinie 2001/29/EG, Art. 14 Abs. 2 Pauschalreiserichtlinie 2015/2302/EU und Art. 82 Datenschutz-Grundverordnung.

66 EuGH, Rs. C-168/00 (Leitner), ECLI:EU:C:2002:163, Slg. 2002, I-02631; nunmehr ausdrücklich hervorgehoben in Erwägungsgrund 34 der Pauschalreiserichtlinie 2015/2302/EU. Ein weiteres Beispiel für immateriellen Schadensersatz auf der Grundlage einer Richtlinie findet sich in Art. 13 Abs. 1 UAbs. 2 Buchst. a) der Durchsetzungsrichtlinie 2004/48/EG.

67 Dazu EuGH, Rs. C-203/99 (Veedfald), ECLI:EU:C:2001:258, Slg. 2001, I-03569, Rn. 27.

68 Vgl. H. Weyer, Schadensersatzansprüche gegen Private kraft Gemeinschaftsrecht, ZeuP 2003, S. 318, 326; M. Ebers (Fn. 23), S. 190 ff.

69 So der BGH zu $§ 33$ Abs. 4 GWB 2005 (BGH, WRP 2018, 941,Rn. 32 - Grauzementkartell II): „Die präventive Wirkung ist nur die Folge des angestrebten effektiveren Ausgleichs entstandener Kartellschäden“" 
erkennbar: Eine Norm gilt auch deswegen für Private, damit die Integration gefördert wird, ${ }^{70}$ und nicht nur deswegen, um - etwa aus einer sozialstaatlichen oder gerechtigkeitstheoretischen Erwägung hinaus - eine Partei, beispielsweise den Verbraucher als „die schwächere Partei“, zu schützen. ${ }^{71}$ Daraus folgt, dass Ansprüche nach dem europäischen Privatrecht zwangsläufig auch der Verhaltenssteuerung dienen. ${ }^{72}$

Auf das konstitutive Element eines europäischen Schadensersatzanspruchs hat die Funktion der Förderung einer EU-policy zunächst keinen Einfluss. Das „Ob“ des Schadensersatzes kann in das Ermessen der Mitgliedstaaten gestellt bleiben, solange sie überhaupt eine Sanktion vorsehen. ${ }^{73}$ Die Funktion, eine politische Zielsetzung zu fördern, beeinflusst jedoch, wie ein Schadensersatzanspruch auszugestalten ist, d.h. das exekutiv-prozedurale Element: Es gelten der Äquivalenz- und insbesondere der Effektivitätsgrundsatz. Nach dem Äquivalenzgrundsatz dürfen die nationalen Vorschriften die Modalitäten der Rechtdurchsetzung nicht weniger günstig ausgestalten als bei gleichartigen Sachverhalten nach innerstaatlicher Art, und nach dem Effektivitätsgrundsatz dürfen die nationalen Vorschriften die Ausübung der durch das Unionsrecht verliehenen Rechte nicht praktisch unmöglich machen oder übermäßig erschweren. ${ }^{74}$ Aus dem Effektivitätsgrundsatz folgt: Wenn die Mitgliedstaaten Schadensersatz vorsehen, dann müssen die Schadensersatzsummen „effektiv“, d.h. ausreichend hoch sein. In diesem Zusammenhang ist die Antidiskriminierungsrechtsprechung des EuGH der 1980er und 1990er Jahre zu verorten.

70 H. Rösler, Schutz des Schwächeren im Europäischen Vertragsrecht - Typisierte und individuelle Unterlegenheit im Mehrebenenprivatrecht, RabelsZ 73 (2009), S. 889, 910; J.-U. Franck (Fn. 29), S. 171;A. Hellgardt(Fn. 20), S. 175 ff., 187 ff. Siehe z.B. Erwägungsgrund 5 der Pauschalreiserichtlinie 2015/2302/EU: „Um einen echten Binnenmarkt für Verbraucher bei Pauschalreisen und verbundenen Reiseleistungen zu schaffen, müssen die Rechte und Pflichten, die sich aus Pauschalreiseverträgen und verbundenen Reiseleistungen ergeben, so harmonisiert werden, dass ein ausgewogenes Verhältnis zwischen einem hohen Verbraucherschutzniveau und der Wettbewerbsfähigkeit der Unternehmen dieser Branche gewährleistet ist.“

71 Art. 169 Abs. 1 AEUV und Art. 38 GRC begründen keine eigenständige Kompetenzgrundlage der EU für verbraucherschützende Gesetzgebung, sondern nur eine ergänzende Zielbestimmung für die Privatrechtssetzung (B. Heiderhoff(Fn. 15), Rn. 13 m.w.N.; H. C. Grigoleit, Der Verbraucheracquis und die Entwicklung des Europäischen Privatrechts, AcP 210 (2010), S. 354, 364 ff.; siehe z.B. Erwägungsgrund 3 der Pauschalreiserichtlinie 2015/2302/EU).

72 Vgl. J.-U. Franck (Fn. 29), S. 186 f.; siehe beispielsweise Erwägungsgrund 9 der Fluggastrechte-Verordnung 261/2004/EG; Erwägungsgrund 6 der Pauschalreiserichtlinie 2015/2302/EU.

73 EuGH, Rs. 14/83 (von Colson und Kamann), ECLI:EU:C:1984:153, Slg. 1984, 01891, Rn. 18; H. Weyer(Fn. 68), S. 328; B. Heiderhoff(Fn. 15), Rn. 292; A. Hellgardt(Fn. 20), S. 187 ff. Siehe etwa Art. 23 Verbraucherrechterichtlinie 2011/83/EU.

74 Grundlegend EuGH, Rs. 33/76 (Rewe), ECLI:EU:C:1976:188, Slg. 1976, 01989, Rn. 5; Rs. 45/76 (Comet), ECLI:EU:C:1976:191, Slg. 1976, 02043, Rn. 11/18; dazu etwa EuGH, Rs. C-453/99 (Courage/Crehan), ECLI:EU:C:2001:465, Slg. 2001, I-06297, Rn. 29; verb. Rs. C-295/04 bis C-298/04 (Manfredi), ECLI:EU:C:2006:461, Slg. 2006, I-06619, Rn. 62; Rs. C-360/09 (Pfleiderer), ECLI:EU:C:2011:389, Slg. 2011, I-05161, Rn. 24; Rs. C-536/11 (Donau Chemie), ECLI:EU:C:2013:366, Rn. 27; Rs. C-557/12 (Kone), ECLI:EU:C:2014:1317, Rn. 22; Rs. C-219/15 (Schmitt/TÜV Rheinland), ECLI:EU:C:2017:128, Rn. 59. 
cc) Rechtsdurchsetzungsfunktion

Eine Anspruchsgrundlage des europäischen Privatrechts kann darüber hinaus aber auch eine dritte Funktion erfüllen, nämlich einen Verstoß gegen das EU-Recht selbst zu sanktionieren, zukünftige Verstöße zu verhindern und damit das EU-Recht durchzusetzen. Der Schwerpunkt liegt hier daher nicht mehr auf der Durchsetzung eines Rechts des Anspruchsinhabers, sondern auf der Durchsetzung europarechtlich begründeter Pflichten durch den Anspruchsgegner. ${ }^{75}$ Kompensation des Geschädigten ist danach nicht das erstrebte Ziel, sondern Mittel zum Zweck. ${ }^{76}$ Der angestrebte Effekt eines solchen Anspruchs ist Prävention durch Verhaltenssteuerung, insbesondere durch Abschreckung. Anders als bei Ansprüchen, die lediglich eine Kompensationsfunktion erfüllen, bestimmt hier das erstrebte Maß an Prävention den Umfang des Schadensausgleichs, nicht umgekehrt. ${ }^{77}$

Kommt einem privatrechtlichen Anspruch auch Rechtsdurchsetzungsfunktion zu, dann wirkt sich dies sowohl auf das konstitutive als auch auf das exekutiv-prozedurale Element aus. In konstitutiver Hinsicht muss das Instrument der Rechtsdurchsetzung, d.h. der Anspruch, durch das Europarecht selbst vorgegeben sein. Regelmäßig dürfte es sich um einen Anspruch auf Schadensersatz handeln, ${ }^{78}$ denkbar ist aber auch ein Anspruch auf Unterlassung oder Beseitigung. Das „Ob“ eines bestimmten Anspruchs ist damit nicht mehr in das Ermessen der Mitgliedstaaten gestellt.

Auch das exekutiv-prozedurale Element ist erheblich durch das Europarecht prädeterminiert. Eine Anspruchsgrundlage, die der Durchsetzung des Europarechts dient, setzt zumeist allein voraus, dass die verletzte Norm dem Einzelnen subjektive Rechte verleiht, ${ }^{79}$ und dass das verbotene Verhalten einen Schaden verursacht hat. Einer Verletzung einer einschränkenden

75 So bereits H. Weyer (Fn. 68), S. 334 ff.

76 D. Poelzig(Fn. 25), S. 172; A. Fuchs, in: Remien (Hrsg.), Schadensersatz im europäischen Privat- und Wirtschaftsrecht, 2012, S. 55, 56 f.; C. Palzer, Transparenz-VO und private Kartellrechtsdurchsetzung Abschied von einem Hoffnungsträger?, ZEuP 2015, S. 416, 422; M. Ebers (Fn. 23), S. 106. Siehe auch BVerfGNJW 2014, 1581,Rn. 22 - Kronzeugenanträge: „Werden wie im vorliegenden Fall Schadensersatzansprüche wegen Verstößen gegen das Kartellverbot geltend gemacht, trägt dies zur wirksamen Kartellbekämpfung bei und steht damit, wie auch der Europäische Gerichtshof anerkannt hat [...] im öffentlichen Interesse der Europäischen Union. “"

77 G. Wagner (Fn. 16), S. 469 f.

78 Vgl. A. Hellgardt(Fn. 20), S. 200; J.-U. Franck (Fn. 29), S. 193 ff.

79 EuGH, Rs. C-253/00 (Muñoz), ECLI:EU:C:2002:497, Slg. 2002, I-07289, Rn. 27; so bereits GA van Gerven, Schlussanträge v. 27.10.1993 in der Rs. C-128/92 (Banks), Rn. 36. Vgl. auch EuGH, Rs. C-219/15 (Schmitt/TÜV Rheinland), ECLI:EU:C:2017:128, Rn. 50, wobei die in diesem Fall einschlägige Medizinprodukterichtlinie 93/42/EWG einen Anspruch gegen die vom Hersteller zur Kontrolle eines Medizinprodukts benannte Stelle gerade nicht vorsah (ebd.,Rn. 59). 
Schutznorm bedarf es demgegenüber nicht. ${ }^{80}$ In Courage brachte der EuGH dies dadurch zum Ausdruck, dass ,jedermann“ Ersatz des Schadens verlangen darf, der ihm durch das wettbewerbswidrige Verhalten entstanden ist. ${ }^{81}$ Sowohl in persönlicher als auch in sachlicher Hinsicht sind sämtliche Schäden zu ersetzen, d.h. materielle und immaterielle Schäden, der entgangene Gewinn, einschließlich die Zahlung von Zinsen. ${ }^{82}$ Defizite beim Schadensausgleich haben nämlich immer auch Präventions- und damit Rechtsdurchsetzungsdefizite zur Folge. ${ }^{83}$ Schwierigkeiten, die Schadenshöhe zu beziffern, müssen zu Lasten des Schädigers gehen, etwa durch Pauschalierung und Beweislastumkehr. ${ }^{84}$ Weder begründet noch erfordert die Rechtsdurchsetzungsfunktion indessen einen Strafschadensersatz. ${ }^{85}$ Der EuGH hat mehrfach darauf hingewiesen, dass die innerstaatlichen Gerichte dafür Sorge tragen dürfen, dass der Schutz der unionsrechtlich gewährleisteten Rechte nicht zu einer ungerechtfertigten Bereicherung der Anspruchsberechtigten führt. ${ }^{86}$ Ein besonderer Schadensersatz wie der Strafschadensersatz muss nur dann aufgrund des Äquivalenzgrundsatzes gewährt werden, wenn er im Rahmen vergleichbarer, auf das innerstaatliche Recht gegründeter Klagen zugesprochen werden kann. ${ }^{87}$

Die Rechtsdurchsetzungsfunktion tritt in der Praxis nie isoliert auf. Die Frage darf daher nicht lauten, ob ein privatrechtlicher Anspruch nach EU-Recht der Kompensation oder der

${ }^{80}$ G. Wagner (Fn. 16), S. 416; R. Nazzini, The Objective of Private Remedies in EU Competition Law, Global Competition Litigation Review 4 (2011), S. 131, 139 f.; A. Hellgardt(Fn. 20), S. 202 f.; J.-U. Franck (Fn. 29), S. 205.Dies unterscheidet die Rechtsdurchsetzung mittels des Privatrechts von der europarechtlichen Staatshaftung, die einen „hinreichend qualifizierten Verstoß“" verlangt (EuGH, verb. Rs. C-46/93 und C48/93 (Brasserie du pechêur und Factortame), ECLI:EU:C:1996:79, Slg. 1996, I-01029,Rn. 51).

81 EuGH, Rs.C-453/99 (Courage/Crehan), ECLI:EU:C:2001:465, Slg. 2001, I-06297, Rn. 26; bestätigt in verb. Rs. C-295/04 bis C-298/04 (Manfredi), ECLI:EU:C:2006:461, Slg. 2006, I-06619, Rn. 60; Rs. C-199/11 (Otis), ECLI:EU:C:2012:684, Rn. 41; Rs. C-536/11 (Donau Chemie), ECLI:EU:C:2013:366, Rn. 21; Rs. C557/12 (Kone), ECLI:EU:C:2014:1317, Rn. 21.

82 EuGH, verb. Rs. C-295/04 bis C-298/04 (Manfredi), ECLI:EU:C:2006:461, Slg. 2006, I-06619, Rn. 95; Rs. C-536/11 (Donau Chemie), ECLI:EU:C:2013:366, Rn. 24; vgl. auch aus der Antidiskriminierungsrechtsprechung EuGH, Rs. C-407/14 (Camacho), ECLI:EU:C:2015:831,Rn. 37.

${ }^{83}$ G. Wagner (Fn. 16), S. 469; C. Vollrath, Das Maßnahmenpaket der Kommission zum wettbewerbsrechtlichen Schadenersatzrecht, NZKart 2013, S. 434, 436; A. Hellgardt(Fn. 20), S. 207.

${ }^{84}$ G. Wagner (Fn. 16), S. 460.

85 Vgl. EuGH, Rs. C-407/14 (Camacho), ECLI:EU:C:2015:831,Rn. 40 (zur Antidiskriminierung); G. Wagner (Fn. 16), S. 476; G. Meeßen(Fn. 63), S. 82 ff.; C. Vollrath(Fn. 83), S. 436; H. Koziol, in: Remien (Hrsg.), Schadensersatz im europäischen Privat- und Wirtschaftsrecht, 2012, S. 5, 13 f.; A. Fuchs(Fn. 76), S. 78; M. Ebers (Fn. 23), S. $110 \mathrm{ff}$.

86 EuGH, Rs. 238/78 (Ireks-Arkady), ECLI:EU:C:1979:226, Slg. 1979, 02955, Rn. 14; verb. Rs. C-441/98 und C-442/98 (Michaïlidis), ECLI:EU:C:2000:479, Slg. 2000, I-07145, Rn. 31; Rs.C-453/99 (Courage/Crehan), ECLI:EU:C:2001:465, Slg. 2001, I-06297, Rn. 30; verb. Rs. C-295/04 bis C-298/04 (Manfredi), ECLI:EU:C:2006:461, Slg. 2006, I-06619, Rn. 94.

87 EuGH, verb. Rs. C-295/04 bis C-298/04 (Manfredi), ECLI:EU:C:2006:461, Slg. 2006, I-06619, Rn. 93. 
Durchsetzung des EU-Rechts dient. ${ }^{88}$ Schadensersatzansprüche erfüllen niemals nur die Rechtsdurchsetzungsfunktion, sondern müssen immerauch dem Ausgleich und der Förderung des Binnenmarktes dienen. ${ }^{89}$ Es ist zudem keine binäre Feststellung, $o b$ ein Schadensersatzanspruch auch der Rechtsdurchsetzung dient oder nicht, sondern vielmehr, inwieweit dies der Fall ist. Dies ist anhand der eben dargestellten Kriterien in jedem Einzelfall zu prüfen.

\section{Das Beispiel der Schadensersatzrichtlinie 2014/104/EU}

Zwar hielt es das Europäische Parlament bereits im Jahr 1961 für „unerlässlich“, die Frage des Schadenersatzes bei Verstößen gegen (damals) Art. 85 und 86 EWG-Vertrag einheitlich zu regeln, ${ }^{90}$ und auch die Europäische Kommission wies in mehreren Dokumenten darauf hin, dass Unternehmen stärker darauf achteten, nicht gegen das Wettbewerbsrecht zu verstoßen, wenn sie den betroffenen Personen Schadensersatz zu leisten hätten. ${ }^{91}$ Weder die Erste Durchführungsverordnung zu den Art. 85 und 86 des EWG-Vertrages ${ }^{92}$ noch die nunmehr gültige Verordnung $1 / 2003^{93}$ sahen indessen eine entsprechende Regelung vor. Erst im Jahr 2014 und damit in Folge der Courage- und Manfredi-Rechtsprechung des EuGH trat die Schadensersatzrichtlinie in Kraft.

Nach Art. 3 Abs. 1 der Richtlinie ${ }^{94}$ gewährleisten die Mitgliedstaaten, dass jede natürliche oder juristische Person, die einen durch eine Zuwiderhandlung gegen Art. 101 oder

88 In dieseRichtungaber $P$. Nebbia, Damages actions for the infringement of EC competition law: compensation or deterrence?European Law Review 33 (2008), S. 23 ff.; wiehierC. Vollrath (Fn. 83), S. 435.

89 Ungenau daher H. Weyer (Fn. 68), S. 333: ,solche Rechte [...], die dem Einzelnen nur deshalb zuerkannt werden, weil sie die effektive Durchsetzung der durch die unmittelbar wirksame Vorschrift begründeten Pflichten fördern“" (Kursivdruck hinzugefügt).

90 Erwägungsgrund 11 der Entschließung des Europäischen Parlaments in Beantwortung der vom Ministerrat der EWG zu dem Vorschlag einer ersten Durchführungsverordnung zu den Artikeln 85 und 86 des EWGVertrages vom Parlament erbetenen Konsultation, ABl. EG 1961, S. 1409.

91 Europäische Kommission, Dreizehnter Bericht über die Wettbewerbspolitik, 1983, Rn. 217 f.; Bekanntmachung über die Zusammenarbeit zwischen der Kommission und den Gerichten der Mitgliedstaaten bei der Anwendung der Artikel 85 und 86 des EWG-Vertrages (93/C 39/05), ABl.EG 1993 C 39/6, Rn. 16; Bekanntmachung über die Behandlung von Beschwerden durch die Kommission gemäß Artikel 81 und 82 EG-Vertrag, ABl.EU 2004 C 101/65, Rn. 13.

92 ABl. EG 1962, S. 204.

93 Siehe allein Erwägungsgrund 7 der Verordnung: „Die einzelstaatlichen Gerichte erfüllen eine wesentliche Aufgabe bei der Anwendung der gemeinschaftlichen Wettbewerbsregeln. In Rechtsstreitigkeiten zwischen Privatpersonen schützen sie die sich aus dem Gemeinschaftsrecht ergebenden subjektiven Rechte, indem sie unter anderem den durch die Zuwiderhandlung Geschädigten Schadenersatz zuerkennen. Sie ergänzen in dieser Hinsicht die Aufgaben der einzelstaatlichen Wettbewerbsbehörden. Ihnen sollte daher gestattet werden, die Artikel 81 und 82 des Vertrags in vollem Umfang anzuwenden.“

94 In deutsches Recht umgesetzt durch § 33a Abs. 1 i.V.m. § 33 Abs. 1 GWB. 
102 AEUV oder gegen nationales Wettbewerbsrecht ${ }^{95}$ verursachten Schaden erlitten hat, den vollständigen Ersatz dieses Schadens verlangen und erwirken kann. Damit nimmt die Vorschrift den acquis aus Courage und Manfredi auf.

Dass die Richtlinie damit dem Schutz subjektiver Rechte, nämlich dem Ausgleich eines entstandenen Schadens dient (im Sinne der Kompensationsfunktion), bedarf keiner weiteren Ausführungen. ${ }^{96}$ Die Richtlinie ist gestützt auf Art. 103 und 114 AEUV und muss damit der Förderung des Binnenmarktes dienen, insbesondere ${ }^{97}$ der „Verwirklichung der in den Artikeln 101 und 102 [AEUV] niedergelegten Grundsätze“‘.98

\section{Bekenntnis der Richtlinie zur privaten Rechtsdurchsetzung}

Es bleibt die Frage: Inwieweit dient die Richtlinie - über die europarechtlich fest verankerte und seit 1998 auch vom deutschen Gesetzgeber anerkannte „Privatisierung des Kartellrechts“"99 hinaus - auch der privaten Durchsetzung des Europarechts selbst? Die Funktion der ,privaten Rechtsdurchsetzung“ ist ausdrücklich in Erwägungsgrund 3 und Art. 1 Abs. 2 der Richtlinie genannt. Sie wird in einem „Instrumentenmix“ zur Durchsetzung des Wettbewerbsrechts positioniert, der sich aus Schadensersatz, einvernehmlicher Streitbeilegung und öffentlicher Rechtsdurchsetzung zusammensetzt. ${ }^{100}$ Eindeutiger praktischer Schwerpunkt des Anwendungsbereichs von wettbewerbsrechtlichen Schadensersatzklagen sind dabei Follow-on-Klagen, d.h. Klagen, die im Nachgang eines

95 Zur Definition des „,nationalen Wettbewerbsrechts“ Art. 2 Nr. 3 Schadensersatzrichtlinie; zur Einbeziehung des nationalen Wettbewerbsrechts in die Richtlinie Erwägungsgrund 10; C. Vollrath (Fn. 83), S. 436.

96 Siehe auch Erwägungsgrund 3: „In Rechtsstreitigkeiten zwischen Privatpersonen schützen sie die sich aus dem Unionsrecht ergebenden subjektiven Rechte, indem sie beispielsweise den durch Zuwiderhandlungen Geschädigten Schadensersatz zuerkennen.“"

97 Der Zusammenhang von Binnenmarkt und Wettbewerb wird in Art. 3 Abs. 1 Buchst. b) AEUV bestätigt, wonach der EU die ausschließliche Zuständigkeit für die Festlegung der „für das Funktionieren des Binnenmarktes erforderlichen Wettbewerbsregeln“" zusteht.

98 Erwägungsgrund 34 der Schadensersatzrichtlinie; siehe auch Art. 1 Abs. 1 Satz 2 sowie Erwägungsgründe 7 bis 10 und 54 der Schadensersatzrichtlinie.

99 Dazu K. Schmidt, „Privatisierung“ des Europakartellrechts - Aufgaben, Verantwortung und Chancen der Privatrechtspraxis nach der VO Nr. 1/2003, ZEuP 2004, S. 881; K. Schmidt, Wirtschaftsrecht: Nagelprobe des Zivilrechts - Das Kartellrecht als Beispiel, AcP 206 (2006), S. 169, 174 ff.; K. Schmidt, Gesetzliches Kartell-Zivilprozessrecht. Der mühsame Weg der $\S \S 87$ ff. GWB aus einem Kartell-Prozessrecht von Gestern zum „Private Enforcement“ für Heute und Morgen, ZWeR 2007, S. 394, 397.

100 Erwägungsgrund 5 der Schadensersatzrichtlinie. 
behördlichen Verfahrens ergangen sind, und nicht Stand-alone-Klagen, bei denen das wettbewerbswidrige Verhalten erst vom Gericht festgestellt werden muss. ${ }^{101}$

Auffällig ist, dass die Richtlinie den vom EuGH in der Antidiskriminierungsrechtsprechung geprägten Begriff der „Abschreckung“ nur im Zusammenhang mit Sanktionen erwähnt, die Gerichte für die Weigerung der Offenlegung von Beweismitteln verhängen dürfen, ${ }^{102}$ nicht jedoch im Hinblick auf die Funktion des Schadensersatzanspruchs selbst. Der Begriff der „Abschreckung“ wird in der jüngeren EU-Rechtsetzung allerdings - soweit ersichtlich lediglich im Zusammenhang mit Geldbußen und sonstigen öffentlich-rechtlichen Sanktionen verwendet, etwa in der Pauschalreiserichtlinie 2015/2302/EU und in der DatenschutzGrundverordnung 2016/679/EU. ${ }^{103}$ Auch der EuGH verwendete in Courage und Manfredi nicht den Begriff der „Abschreckung“, was wiederum die These von der Diskontinuität mit der Antidiskriminierungsrechtsprechung bestätigt. Dies ist offenbar darauf zurückzuführen, dass der Begriff der „Abschreckung“ im Zusammenhang mit zivilrechtlichen Ansprüchen in einer negativen Konnotation mit Strafschadensersatz steht. Erwägungsgrund 32 der Rom IIVO bringt dies zum Ausdruck. Darin heißt es, dass ein ,unangemessener, über den Ausgleich des entstandenen Schadens hinausgehender Schadensersatz mit abschreckender Wirkung oder Strafschadensersatz" als mit dem ordre publicunvereinbar angesehen werden darf.

Den Begriff der „Abschreckung“ ist daher für die vorliegende Untersuchung nicht in Zusammenhang mit punitive damages, sondern lediglich als präventives Instrument der Verhaltenssteuerung, hier eben durch einen umfassenden Schadensersatzanspruch, zu verstehen. ${ }^{104}$ Dass die Richtlinie in Art. 3 Abs. 3 Strafschadensersatz ausschließt, steht damit ihrer möglichen Rechtsdurchsetzungsfunktion nicht entgegen. Die entscheidende Frage ist vielmehr, inwieweit die Richtlinie gemäß den oben aufgeführten Kriterien in der Sache

101 G. Meeßen(Fn. 63), S. 144 ff.; H. Schweitzer, Die neue Richtlinie für wettbewerbsrechtliche Schadensersatzklagen, NZKart 2014, S. 335, 345; R. Inderst/S. Thomas, Schadensersatz bei Kartellverstößen, 2. Aufl. 2018, S. 100; M. C. Lucey, EU competition law Damages Directive: recalibrating the equilibrium between private and public enforcement?, Journal of Business Law 2018, S. 390, 400 f.; A. Stephan (Fn. 25), S. 154.Dies folgt mittelbar auch aus Art. 9 Schadensersatzrichtlinie.

102 Art. 8 Abs. 2 Schadensersatzrichtlinie.

103 Siehe z.B. Art. 83 Abs. 1 und Abs. 9 sowie Erwägungsgründe 151 und 152 der DatenschutzGrundverordnung 2016/679/EU, Art. 25 und Erwägungsgrund 47 der Pauschalreiserichtlinie 2015/2302/EU, Art. 8 Abs. 2 und Erwägungsgrund 33 der Schadensersatzrichtlinie.

104 Vgl. BGHZ 190, 145,Rn. 62 - ORWI;RegE, BT-Drucks 15/3640, S. 35; D. Poelzig(Fn. 25), S. 157, $172 ;$ S. J. Geibel, Die Richtlinie über die Haftung für Kartellrechtsverstöße: Förderung der Privatinitiative zur Durchsetzung des Rechts und Eckstein für ein Europäisches Schadensrecht? in: Stumpf/Kainer/Baldus (Hrsg.), Privatrecht, Wirtschaftsrecht, Verfassungsrecht. Privatinitiative und Gemeinwohlhorizonte in der europäischen Integration, Festschrift für Peter-Christian Müller-Graff zum 70. Geburtstag am 29. September 2015, 2015, S. 558, 563 f.; C. Brömmelmeyer, Die Ermittlung des Kartellschadens nach der Richtlinie 2014/104/EU, NZKart 2016, S. 2, 4; T. Lettl, Kartellrecht, 4. Aufl. 2017, § 11,Rn. 80. 
„abschreckend“ im Sinne einer Verhaltenssteuerung ist, die auf die Durchsetzung des Unionsrechts abzielt.

$\mathrm{Zu}$ unterscheiden ist insofern zwischen dem konstitutiven Element des Schadensersatzanspruchs einerseits und seinem exekutiv-prozeduralen Element andererseits.

\section{Konstitutives Element}

Die Richtlinie überlässt das konstitutive Element nicht dem Ermessen der Mitgliedstaaten, sondern gibt das „Ob“ eines Schadensersatzanspruchs unmittelbar vor. Dasist ein Indiz dafür, dass der Anspruch auch der Durchsetzung des Unionsrechts selbst dient. Erwägungsgrund 3 der Richtlinie unterstreicht dies, indem er besagt, dass die volle Wirksamkeit der Art. 101 und 102 AEUV einen Schadensersatzanspruch „erforder[t]“.

\section{Exekutiv-prozedurales Element}

Damit ist noch zu prüfen, inwieweit die Richtlinie das exekutiv-prozedurale Element zur Rechtsdurchsetzung prädeterminiert. Ausgangspunkt der Richtlinie ist die Verfahrensautonomie der Mitgliedstaaten. ${ }^{105}$ Auf die allgemeine Einschränkung der Rechtsbehelfsgarantien der Art. 19 Abs. 1 UAbs. 2 EUV und Art. 47 Abs. 1 GRC weist Erwägungsgrund 4 der Schadensersatzrichtlinie hin. Art. 4 Schadensersatzrichtlinie wiederholt zudem den Effektivitäts- und Äquivalenzgrundsatz, aber auch dies ist noch kein Indiz dafür, dass die Richtlinie auch der Durchsetzung des Europarechts dient, da dieses Gebot bereits - wie eben dargestellt ${ }^{106}$ - aus der Binnenmarktrelevanz folgt. Die entscheidende Frage ist daher, inwieweit die Richtlinie selbst ${ }^{107}$ den Schadensersatzanspruch in einer Weise ausgestaltet, dass der Anspruch der Durchsetzung des Unionsrechts dient.Drei Komponenten werden hier beispielhaft für diese Analyse herangezogen: der Umfang des

\footnotetext{
105 Siehe Erwägungsgrund 11.

106 Siehe II.3.b)bb).

107 Irreführend ist daher der erste Satz des Erwägungsgrundes 11: „Da keine entsprechenden unionsrechtlichen Vorschriften bestehen, gelten für Schadensersatzklagen die innerstaatlichen Vorschriften und Verfahren der Mitgliedstaaten." Die Richtlinie legt allerdings selbst unionsrechtliche Vorschriften fest, die innerstaatliche Schadensersatzklagen prägen, beispielsweise die Regelung zur Verjährung in Art. 10. Zutreffend wäre daher gewesen, statt „Da“ das Wort „Soweit“ zu verwenden. Gleiches gilt für Erwägungsgrund 46 zur Ermittlung des Schadensumfangs: „Da keine unionsrechtlichen Vorschriften über die Ermittlung des Umfangs eines durch eine Zuwiderhandlung gegen das Wettbewerbsrecht verursachten Schadens bestehen, [...].“. Auch hier wäre das Wort „Soweit“ angezeigt gewesen.
} 
Schadensersatzanspruchs, die Aktivlegitimation und Durchsetzbarkeit sowie die Frage der Zurechnung. ${ }^{108}$

\section{a) Umfang des Schadensersatzanspruchs}

Unter klinischen Bedingungen wäre ein Anspruch, der auch der Durchsetzung des Europarechts dient, auf umfassenden Schadensersatz gerichtet. Denn Defizite beim Schadensausgleich haben immer auch Präventions- und damit Rechtsdurchsetzungsdefizite zur Folge. ${ }^{109}$

Die Richtlinie spricht an mehreren Stellen davon, dass „vollständiger Ersatz“ zu leisten ist. ${ }^{110}$ Art. 3 Abs. 2 Schadensersatzrichtlinie bestimmt insoweit mittels der Differenzmethode, ${ }^{111}$ dass der ,,vollständige Ersatz“ den Geschädigten in die Lage versetzt, in der er sich befunden hätte, wenn die Zuwiderhandlung gegen das Wettbewerbsrecht nicht begangen worden wäre. ${ }^{112}$ Der ,vollständige Ersatz“ erfasst daher das Recht auf Ersatz der eingetretenen Vermögenseinbuße und des entgangenen Gewinns, ${ }^{113}$ zuzüglich der Zahlung von Zinsen. ${ }^{114}$

Um seine abschreckende Wirkung vollumfänglich zu entfalten, müssten Schwierigkeiten, den Schaden zu beziffern, zu Lasten des Schädigers gehen, etwa durch Pauschalierung, Beweislastumkehr und Schätzungen. Gerade bei der Haftung für Kartellverstöße gestaltet sich die Ermittlung des Schadensumfangs häufig sehr schwierig, weshalb die Europäische Kommission den nationalen Gerichten einige Handreichungen gegeben hat. ${ }^{115}$ Aber auch in

${ }^{108}$ Die Untersuchung ließe sich auf die weiteren Komponenten der Richtlinie erstrecken, etwa die Offenlegung von Beweismitteln (Art. 5 ff.) und die Verjährung (Art. 10).

109 Siehe unter II.3.b)cc).

110 Siehe Art. 1, 3, 4, 11, 12 und 20 Abs. 2 Buchst. a) sowie Erwägungsgründe 13, 38, 44 und 51.

111 C. Brömmelmeyer (Fn. 104), S. 2.

112 Siehe auch Art. 2 Nr. 20 der Richtlinie: „Preisaufschlag“ ist danach „die Differenz zwischen dem tatsächlich gezahlten Preis und dem Preis, der sich ohne die Zuwiderhandlung gegen das Wettbewerbsrecht ergeben hätte“.

113 Siehe auch Art. 12 Abs. 3.

114 Siehe Erwägungsgrund 12.

115 Europäische Kommission, Mitteilung der Kommission zur Ermittlung des Schadensumfangs bei Schadensersatzklagen wegen Zuwiderhandlungen gegen Artikel 101 oder 102 des Vertrags über die Arbeitsweise der Europäischen Union, 2013/C 167/07, ABl. 1967 C 167/19; Praktischer Leitfaden zur Ermittlung des Schadensumfangs bei Schadensersatzklagen im Zusammenhang mit Zuwiderhandlungen gegen Artikel 101 oder 102 des Vertrages über die Arbeitsweise der Europäischen Union, abrufbar unter ec.europa.eu/competition/antitrust/actionsdamages/quantification_guide_de.pdf. Siehe zur Ermittlung von Kartellschäden auch U. Brunner/P. Y. Bacher, Ermittlung von Kärtellschäden für die zivilrechtliche Geltendmachung - Eine Gebrauchsanweisung, NZKart 2017, S. 345 ff. 
dieser Hinsicht „liefert“ die Richtlinie weitestgehend: Nach Art. 17 Abs. 1 dürfen weder die Beweislast noch das Beweismaß für die Ermittlung des Schadensumfangs den Schadensersatz praktisch unmöglich machen oder übermäßig erschweren. Die nationalen Gerichte müssen stattdessen befugt sein, die Höhe des Schadens zu schätzen. ${ }^{116}$ Nach Art. 17 Abs. 3 kann eine nationale Wettbewerbsbehörde auf Antrag eines Gerichts diesem bei der Festlegung der Höhe des Schadensersatzes behilflich sein. ${ }^{117}$ Den Kartellbehörden kommt damit gleichsam die Rolle eines amicuscuriae $\mathrm{zu}^{118}$

Art. 17 Abs. 2 begründet eine widerlegbare Vermutung für eine Schadensverursachung. ${ }^{119}$ Die Vermutung umfasst das Bestehen eines Schadens ${ }^{120}$ und dessen Verursachung durch den Kartellrechtsverstoß, nicht jedoch die konkrete Höhe des Schadens. ${ }^{121}$ Die Schadensvermutung ist allerdings auf Kartelle i.S.d. Art. 2 Nr. 14 der Richtlinie beschränkt, „da diese durch ihren geheimen Charakter die Informationsasymmetrie verstärken und es dem Kläger erschweren, die für den Nachweis des Schadens erforderlichen Beweise zu beschaffen. “122 Andere Wettbewerbsbeschränkungen als Kartelle, etwa sonstige horizontale Beschränkungen oder der Missbrauch einer marktbeherrschenden Stellung i.S.d. Art. 102 AEUV, sind nicht erfasst. ${ }^{123}$ Dies legt nahe, dass der Richtlinie nur - aber immerhin - im Hinblick auf Kartelle Rechtsdurchsetzungsfunktion zukommt.

\section{b) Aktivlegitimation und Durchsetzbarkeit}

\footnotetext{
${ }^{116}$ Einer expliziten Umsetzung dieser Vorgabe in deutsches Recht bedurfte es nicht, da diese Möglichkeit bereits in $\S 287$ ZPO vorgesehen ist, auf den $\S 33 \mathrm{a}$ Abs. 3 Satz 1 GWB verweist.

${ }^{117}$ Umgesetzt in deutsches Recht durch $\S 90$ Abs. 5 GWB.

${ }^{118}$ D. Poelzig(Fn. 25), S. 198.

119 Umgesetzt durch $\S 33$ a Abs. 2 GWB.

120 A.A. L. Ascheberg, Kartellrechtliche Schadensersatzrichtlinie 2014/104/EU - Neuerungen und Schwierigkeiten bei der Umsetzung in deutsches Recht, JA 2016, S. 1101, 1103.

121 Erwägungsgrund 47; dazu C. Vollrath (Fn. 83), S. 440; H. Schweitzer(Fn. 101), S. 337; C. Brömmelmeyer (Fn. 104), S. 8; T. Makatsch/A. S. Mir, Die neue EU-Richtlinie zu Kartellschadensersatzklagen - Angst vor der eigenen „Courage“?, EuZW 2015, S. 7, 8; G. Klumpe/T. Thiede, Keeping theFloodgatesShut Kartellschadensersatz nach der 9. GWB-Novelle, NZKart 2017, S. 332, 334; A. Fritzsche, Die Schadensvermutung - Auslegungsfragen zum Kartellzivilrecht nach der 9. GWB-Novelle, NZKart 2017, S. 581 ff. Für die Kodifikation eines Mindestschadens bei Kartellen C. Kersting/N. Preuß, Umsetzung der Kartellschadensersatzrichtlinie (2014/104/EU), 2015, Rn. 58 ff.

122 Erwägungsgrund 47.

${ }^{123}$ Kritisch dazu N. Dunne, The Roleof Private Enforcementwithin EU Competition Law, Cambridge Yearbookof European Legal Studies 16 (2014), S. 143, 183 f. Die Mitgliedstaaten dürften aber die Schadensvermutung auf Formen der Wettbewerbsbeschränkung erstrecken.
} 
Die Schadensersatzrichtlinie entfaltet zudem umso größere abschreckende Wirkung, je näher sie einer vollen Kompensation aller Geschädigten kommt. Und tatsächlich gewährt Art. 3 Abs. 1 ,jede[r] natürliche[n] oder juristische[n] Person“ einen Anspruch auf Schadensersatz. Dies korrespondiert mit der Legaldefinition des „Geschädigte[n]“ in Art. 2 Nr. 6 Schadensersatzrichtlinie, nämlich ,jede Person, die einen durch eine Zuwiderhandlung gegen das Wettbewerbsrecht verursachten Schaden erlitten hat". Der Kreis potentieller Anspruchsteller ist damit denkbar weit gezogen. Insbesondere bedarf es lediglich des Eintritts eines Schadens; ein einschränkendes Erfordernis einer Schutznormverletzung sieht die Richtlinie nicht vor. Potentielle Anspruchsinhaber sind damit etwa Kunden von Kartellaußenseitern, die ihre Preise erhöhen (sog. Preisschirmeffekt), ${ }^{124}$ Lieferanten von Einkaufskartellen bzw. von diskriminierenden Marktbeherrschern, ${ }^{125}$ Kartellbeteiligte selbst, ${ }^{126}$ Verbraucher und Behörden. ${ }^{127}$

Art. 3 Abs. 1 verlangt aber nicht nur, dass die betroffene Person Ersatz des Schadens „verlangen“, sondern auch „erwirken“ kann. Die praktische Umsetzung dieses Gebots ist allerdings dann problematisch, wenn sich ein unmittelbar Betroffener mit der in Art. 13 Satz 1 kodifizierten sog. passing on defence konfrontiert sieht, weil er die erhöhten Preise auf die Zweit- oder Endabnehmer abwälzen konnte und somit keinen Schaden erlitten hat. ${ }^{128}$ Art. 12 Abs. 1 besagt ausdrücklich, dass, ,jeder Geschädigte unabhängig davon, ob er unmittelbarer oder mittelbarer Abnehmer ${ }^{129}$ eines Rechtsverletzers ist, Schadensersatz verlangen kann“. ${ }^{130}$ Damit soll allen geschädigten Abnehmern, einschließlich der Verbraucher, dieGeltendmachung von Schadensersatzansprüchen ermöglicht werden. Im Gegensatz zur ORWI-Rechtsprechung des $\mathrm{BGH}^{131}$ muss der Abnehmer allerdings selbst nachweisen, dass der aus der Weitergabe des Preisaufschlags an die nächste Marktstufe resultierende Vorteil nicht durch einen Nachfragerückgang kompensiert worden ist. ${ }^{132}$ Insgesamt schwächen die

\footnotetext{
${ }^{124}$ EuGH, Rs. C-557/12 (Kone), ECLI:EU:C:2014:1317.

${ }^{125}$ F. Stancke, Die Betroffenheit und Aktivlegitimation im Rahmen kartellrechtlicher Schadensersatzklagen, NZKart 2017, S. 636, 640 .

${ }^{126}$ EuGH, Rs. C-453/99 (Courage/Crehan), ECLI:EU:C:2001:465, Slg. 2001, I-06297.

${ }^{127}$ Erwägungsgründe 3 und 13 der Richtlinie.

${ }^{128}$ Zur passing on defence im deutschen Recht zunächst BGHZ 190, 145, Rn. 55 ff. - ORWI und nunmehr § 33c GWB.

${ }^{129}$ Legaldefiniert in Art. 2 Nr. 23 und 24 Schadensersatzrichtlinie.

${ }^{130}$ Siehe auch Erwägungsgrund 13: „Das Recht auf Schadensersatz ist für jede natürliche oder juristische Person [...] anerkannt, ohne Rücksicht darauf, ob eine unmittelbare vertragliche Beziehung zu dem zuwiderhandelnden Unternehmen besteht".

${ }^{131}$ BGHZ 190, 145,Rn. 58 ff. - ORWI.

${ }^{132}$ H. Schweitzer (Fn. 101), S. 339.
} 
passing on-Regelungen der Richtlinie damit den unmittelbaren und stärken den mittelbaren Abnehmer. ${ }^{133}$

Die mittelbaren Abnehmer, insbesondere Verbraucher, haben jedoch regelmäßig keinen vernünftigen Anreiz, ihren Kleinschaden geltend $\mathrm{zu}$ machen. ${ }^{134} \mathrm{Um}$ gleichwohl einen umfassenden Schadensersatz auf der Makroebene zu gewährleisten, müsste die Richtlinie daher entweder eine Bündelung von Einzelansprüchen durch Sammelklagen oder eine Mehrfachentschädigung einzelner Kläger einfordern. ${ }^{135}$ Dies ist indessen nicht der Fall: Zum einen weist der 13. Erwägungsgrund darauf hin, dass die Mitgliedstaaten nicht dazu verpflichtet werden sollten, Verfahren des kollektiven Rechtsschutzes einzuführen. Zum anderen statuiert Art. 12 Abs. 1 ausdrücklich ein Bereicherungsverbot des Klägers, worunter Erwägungsgrund 13 auch die Mehrfachentschädigung subsumiert. Den durch die Richtlinie vorgegebenen Korridor, wonach sowohl eine Überkompensation des Klägers als auch eine Nichthaftung des Rechtsverletzers zu verhindern sind, überschreitet die Richtlinie somit im Falle eines Zielkonflikts zugunsten des Rechtsverletzers. Dies wiederum offenbart ein Bekenntnis der Richtlinie zur bloßen Kompensationsfunktion des Schadensersatzanspruchs und gegen seine Rechtsdurchsetzungsfunktion. Abzuwarten bleiben daher die Bestrebungen der Europäischen Kommission, die Mitgliedstaaten zur Einführung von Instrumenten kollektiver Rechtsdurchsetzung zu bewegen. ${ }^{136}$ Die jüngst in Deutschland beschlossene Musterfeststellungsklage wird dem jedenfalls nicht abhelfen, da Verbraucherverbände danach nur berechtigt sind, das Bestehen von Ansprüchen feststellen zu lassen. ${ }^{137}$ Es bleibt aber dem einzelnen Geschädigten überlassen, seinen individuell erlittenen Schaden zu beziffern und durchzusetzen. $^{138}$ Schließlich ist auch die Aktivlegitimation von Unternehmens- und

${ }^{133}$ T. Makatsch/A. S. Mir(Fn. 121), S. 12; A. Weitbrecht, Eine neue Ära im Kartellschadensersatzrecht - Die 9. GWB-Novelle, NJW 2017, S. 1574, 1575.

${ }^{134}$ D. Poelzig(Fn. 25), S. 138 f.; D. Calisti/L. Haasbeek/F. Kubik, The Directive on Antitrust Damages Actions: Towards a stronger competition culture in Europe, founded on the combined power of public and private enforcement of the EU competition rules, NZKart 2014, S. 466, 468; T. Makatsch/A. S. Mir (Fn. 121), S. 12; L. Ascheberg(Fn. 120), S. 1102; S. Peyer, Compensation and the Damages Directive, European Competition Journal 12 (2016), S. 87, 107 f.; G. Klumpe/T. Thiede(Fn. 121), S. 333; A. Weitbrecht, Kartellschadensersatz 2017, NZKart 2018, S. 106, 108; A. Stephan (Fn. 25), S. 166 f.

135 G. Wagner (Fn. 16), S. 464 ff.; G. Meeßen(Fn. 63), S. 70 f.; S. Peyer(Fn. 134), S. 108; G. Klumpe/T. Thiede(Fn. 121), S.333.

136 Siehe Empfehlung der Kommission vom 11.6.2013: Gemeinsame Grundsätze für kollektive Unterlassungsund Schadensersatzverfahren in den Mitgliedstaaten bei Verletzung von durch Unionsrecht garantierten Rechten (2013/396/EU), ABl.EU 2013 L 201/60, dazu R. Hempel, War da nicht noch etwas? - Zum kollektiven Rechtsschutz im Kartellrecht, NZKart 2013, S. 494 ff.

$137 \S 606$ Abs. 1 Satz 1 ZPO i.d.F. des Gesetzes zur Einführung einer zivilprozessualen Musterfeststellungsklage vom 12.7.2018, BGB1. 2018 I S. 1151. § 606 ZPO tratam 1.11.2018 in Kraft.

${ }^{138}$ C. Rother, Kartellschadensersatz nach der 9. GWB-Novelle, NZKart 2017, S. 1, 2. 
Verbraucherverbänden gemäß § 33 Abs. 4 GWB auf Unterlassungs- und Beseitigungsklagen nach $\S 33$ Abs. 1 GWB sowie Vorteilsabschöpfungsklagen gemäß $\S 34 \mathrm{a}$ GWB beschränkt und umfasst gerade keine Schadensersatzklagen nach $\S 33 a$ GWB. ${ }^{139}$

\section{c) Zurechnung}

Wie für den primärrechtlichen acquisstellt sich für den Anspruch nach Art. 3 Schadensersatzrichtlinie die Frage nach weiteren haftungsbegründenden Tatbestandsmerkmalen, insbesondere Kausalität und Zurechnung. Als exekutiv-prozedurale Elemente ist die Ausgestaltung von Kausalität und Zurechnung zunächst Sache der Mitgliedstaaten, was Erwägungsgrund 11 der Richtlinie bestätigt. Eine Vollharmonisierung liegt daher nicht vor. ${ }^{140}$ Einschränkungen erfährt dieses Ermessen allerdings durch die Rechtsbehelfsgarantie des Art. 19 Abs. 1 UAbs. 2 EUV und Art. 47 Abs. 1 GRC, den Äquivalenzgrundsatz, den Effektivitätsgrundsatz und Festlegungen durch das Europarecht selbst. ${ }^{141}$ Zwei Entscheidungen des EuGH haben bereits vor Erlass der Richtlinie solche Einschränkungen begründet: Zum einen sah der EuGH in Courage in der strengen Anwendung der englischen ,illegalityrule“, wonach eine Partei eines rechtswidrigen Vertrags von der anderen Partei keinen Schadensersatz verlangen kann, einen Verstoß gegen Unionsrecht. ${ }^{142}$ Zum anderen entschied der Gerichtshof in der Rechtssache Kone, dass die Mitgliedstaaten einen „ursächlichen Zusammenhang“ in Fällen sog. Preisschirmeffekte nicht „kategorisch und unabhängig von den speziellen Umständen des konkreten Falles“ ausschließen dürfen, in diesem Fall aufgrund der Rechtsfigur des Schutzzwecks der Norm nach österreichischem Recht. ${ }^{143}$ Indem sie das Ermessen der Mitgliedstaaten bei der Ausgestaltung von Kausalität und Zurechnung einschränken, indizieren diese Entscheidungen somit, dass der kartellrechtliche Schadensersatzanspruch durchaus eine Rechtsdurchsetzungsfunktion besitzt.

\footnotetext{
139 F. Stancke(Fn. 125), S. 642 m.w.N.

${ }^{140}$ So auch C. Vollrath (Fn. 83), S. 439.

141 Siehe EuGH, verb. Rs. C-295/04 bis C-298/04 (Manfredi), ECLI:EU:C:2006:461, Slg. 2006, I-06619, Rn. 64; Erwägungsgrund 11 der Schadensersatzrichtlinie.

${ }^{142}$ EuGH, Rs. C-453/99 (Courage/Crehan), ECLI:EU:C:2001:465, Slg. 2001, I-06297, Rn. 31.

143 EuGH, Rs. C-557/12 (Kone), ECLI:EU:C:2014:1317, Rn. 33. Preisschirmeffekte beschreiben das Phänomen, dass ein Kartellaußenseiter im Windschatten des Kartells seine Preise dem erhöhten Niveau anpasst.
} 


\section{Praktische Konsequenzen am Beispiel der Auslegung des $§ 33$ Abs. 3 GWB}

Die Frage, inwieweit ein Anspruch des europäischen Privatrechts auch der Durchsetzung des Unionsrechts dient, hat erhebliche praktische Auswirkungen. Sie betrifft die teleologische Auslegung der Anspruchsgrundlage und damit die zentrale Auslegungsmethode des EuGH. ${ }^{144}$ Dies beeinflusst auch die europarechtskonforme Auslegung nationalen Rechts, welches der Umsetzung des Anspruchs dient. ${ }^{145}$ An einem Beispiel des GWB ist dies zu verdeutlichen. Nach $\S 33 a$ Abs. 1 i.V.m. § 33 Abs. 1 GWB sind „Betroffene“ zur Geltendmachung eines Schadensersatzanspruchs berechtigt. ${ }^{146} \mathrm{Nach} \S 33$ Abs. 3 GWB ist betroffen, „wer als Mitbewerber oder sonstiger Marktbeteiligter durch den Verstoß beeinträchtigt ist". Andere Marktbeteiligte als Mitbewerber sind Abnehmer oder Anbieter auf der Marktgegenseite. ${ }^{147}$ Nicht erfasst von $\S 33$ Abs. 3 GWB wären danach etwa Gesellschafter und Arbeitnehmer, die durch ein wettbewerbswidriges Verhalten einen wirtschaftlichen Nachteil erleiden. ${ }^{148}$ Der Wortlaut der Richtlinie, der seinerseits auf die „Jedermann“-Formel des EuGH gestützt ist, lässt eine solche Einschränkung auf den ersten Blick allerdings nicht zu. Vielmehr erscheint danach jeder anspruchsberechtigt, der auch nur irgendwie in seinen Interessen beeinträchtigt ist. ${ }^{149}$ Konsequent $\mathrm{zu}$ Ende gedacht, wäre dies mit der Rechtsdurchsetzungsfunktion der Richtlinie auch begründbar.

Indessen erscheint eine Einschränkung der Anspruchsberechtigung rechtspolitisch geboten, um bloß faktische Beeinträchtigungen auszuschließen. ${ }^{150}$ So kann etwa die Schadensvermutung bei Kartellen nicht bedeuten, dass für jeden denkbaren Dritten die

\footnotetext{
${ }^{144}$ M. Göke, Der Einzelne im Spannungsfeld von Teleologie und Deontologie in der Rechtsprechung des EuGH, 2015, S. 7, 122 ff.; M. Ebers (Fn. 23), S. 12; B. Heiderhoff(Fn. 15), Rn. 109 m.w.N.; vgl. EuGH, Rs. 292/82 (Merck), ECLI:EU:C:1983:335, Slg. 1983, 03781, Rn. 12; Rs. 337/82 (St. Nikolaus Brennerei), ECLI:EU:C:1984:69, Slg. 1984, 01051, Rn. 10.

${ }^{145}$ R. Hempel, An der schönen blauen Donau - Akteneinsicht in Kronzeugenanträge im Fluss. Zugleich Besprechung EuGH, Urt. v. 6. 6. 2013 - Rs. C-536/11 - Donau Chemie, ZWeR 2014, S. 203, 219.

146 So die trotz des nicht eindeutigen Wortlauts ganz h.M., z.B. G. Meeßen(Fn. 63), S. 168 ff.; D. Poelzig(Fn. 25), S. 158 f. m.w.N. zur Gegenauffassung; F. Stancke(Fn. 125), S. 636; T. Lettl(Rn. 104), § 11,Rn. 30; A. Weitbrecht(Fn. 134), S. 107; J. Bornkamm/J. Tolkmitt, in: Langen/Bunte (Hrsg.), Kartellrecht, Band 1: Deutsches Kartellrecht, 13. Aufl. 2018, § 33a GWB,Rn. 6.

147 T. Lettl(Rn. 104), § 11,Rn. 42; J. Bornkamm/J. Tolkmitt(Fn. 146), § 33 GWB,Rn. 18; siehe etwa BGH NJW 2019, 661,Rn. 44 - Schienenkartell.

148 A. Görner, Die Anspruchsberechtigung der Marktbeteiligten nach $\S 33$ GWB, 2007, S. 144, 164 f.; D. Poelzig(Fn. 25), S. 159

149 Vgl. G. Meeßen(Fn. 63), S. 196 f.; C. Vollrath (Fn. 83), S. 440; M. Ebers (Fn. 23), S. 592 f.; A. Stephan (Fn. 25), S. 163.

${ }^{150}$ Vgl. M. Ebers (Fn. 23), S. 178 ff., 593 f.; S. Thonig, Privater Rechtsschutz gegen den Missbrauch von Marktmacht, 2016, S. 131; F. Stancke(Fn. 125), S. 641.
} 
Vermutung besteht, dass dieser einen Schaden erlitten hat, und der Beklagte dies widerlegen muss. ${ }^{151}$ Auch sieht die Richtlinie selbst etwas versteckt eine - systemfremde und rechtspolitisch umstrittene ${ }^{152}$ - Einschränkung der „Jedermann“-Formel vor: Gemäß Art. 11 Abs. 2 haftet ein kleines oder mittleres Unternehmen (KMU) unter bestimmten Voraussetzungen nur gegenüber seinen unmittelbaren und mittelbaren Abnehmern.

Dogmatisch ließe sich eine Einschränkung der Anspruchsberechtigung auf Marktteilnehmer, wie $\S 33$ Abs. 3 GWB sie vorsieht, mit dem Gedanken des Schutzzwecks der Norm begründen. ${ }^{153}$ Als Instrument der Zurechnung ist der Schutzzweck der Norm Bestandteil des exekutiv-prozeduralen Elements und daher grundsätzlich Sache der Mitgliedstaaten. Dies bestätigt Erwägungsgrund 11 Satz 5 der Richtline, wonach die Mitgliedstaaten entsprechende Bedingungen für Schadensersatz „wie etwa Zurechenbarkeit, Adäquanz oder Verschulden“ beibehalten dürfen. Dies gilt allerdings nur, „sofern sie mit der Rechtsprechung des Gerichtshofs, dem Effektivitäts- und dem Äquivalenzgrundsatz und den Bestimmungen dieser Richtlinie im Einklang stehen.“ Es ist daher denkbar, dass der EuGH auch diese Einschränkung des Schadensersatzanspruchs durch mitgliedstaatliches Recht - ähnlich wie in der Rechtssache Kone - für europarechtswidrig erklärt. Zur Begründung hierfür könnte der Gerichtshof dann auf die Rechtsdurchsetzungsfunktion des Schadensersatzanspruchs nach der Richtlinie verweisen, wie sie hier nachgewiesen wurde.

${ }^{151}$ A. Fritzsche(Fn. 121), S. 582, unter Verweis auf die Gegenauffassung von C. Kersting, Kartellschadensersatz: Haftungstatbestand - Bindungswirkung- Schadensabwälzung, in: Kersting/Podszun (Hrsg.), Die 9. GWB-Novelle, 2017, Kap. 7,Rn. 56.

152 Siehe z.B. C. Kersting, Die neue Richtlinie zur privaten Rechtsdurchsetzung im Kartellrecht, WuW 2014, S. 564, 567 f.; P. Stauber/H. Schaper, Die Kartellschadensersatzrichtlinie - Handlungsbedarf für den deutschen Gesetzgeber, NZKart 2014, S. 346, 352; S. J. Geibel(Fn. 104), S. 565 Fn. 30; C. Kersting/N. Preuß(Fn. 121), Rn. 121; L. Ascheberg (Fn. 120), S. 1113; M. Ebers (Fn. 23), S. 597.

153 Vgl. OLG Düsseldorf, Urt. v. 2.7.2014, Az. VI-U (Kart) 22/13, Rn. 45 - Telefonauskunftsdienst; G. Meeßen(Fn. 63), S.201 ff.; M. Ebers (Fn. 23), S. 194; S. Thonig(Fn. 150), S. 134; E. Rehbinder, in: Loewenheim/Meessen/Riesenkampff/Kersting/Meyer-Lindemann (Hrsg.), Kartellrecht, 3. Auflage 2016, § 33 GWB,Rn. 16; C. Krohs, in: Busche/Röhling (Hrsg.), Kölner Kommentar zum Kartellrecht, Band 1: Deutsches Kartellrecht, 2017, § 33 GWB,Rn. 126; T. Lettl(Rn. 104), § 11,Rn. 112; J. Bornkamm/J. Tolkmitt(Fn. 146), § 33 GWB,Rn. 19. 